\title{
UDC 62-52
}

SCOPUS CODE 1701

https://doi.org/10.36073/1512-0996-2021-1-75-97

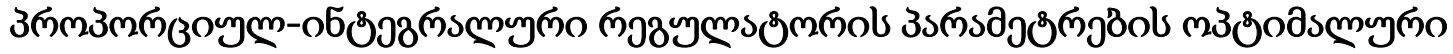

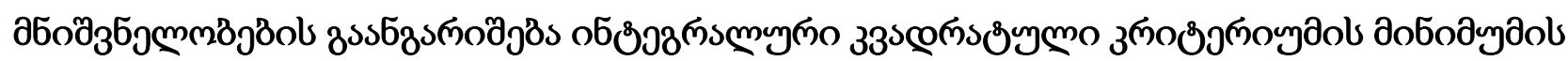

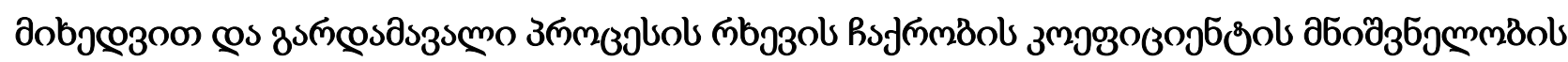

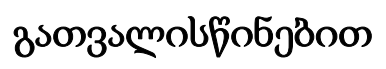

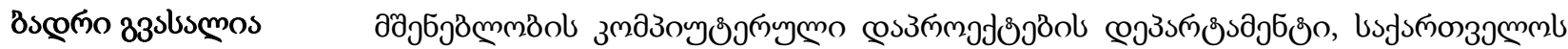

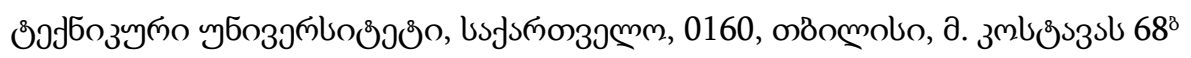
E-mail: Gvasaliabadri01@gtu.ge

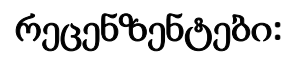

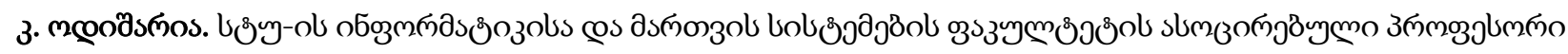
O_korneli@yahoo.com

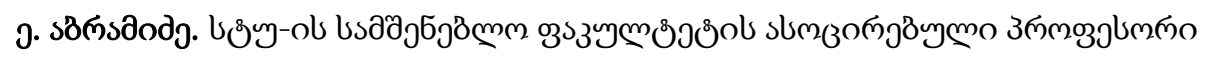

Edisoni.abramidze@mail.ru

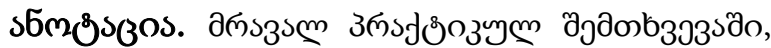

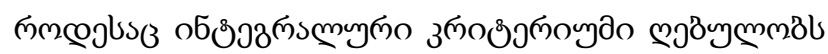

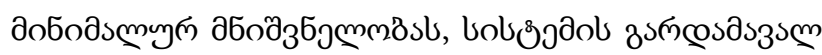

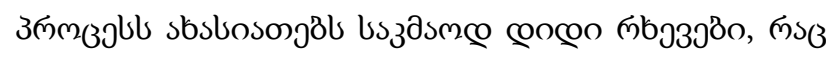

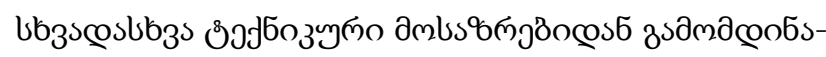

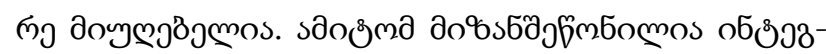

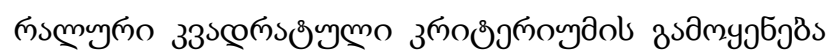

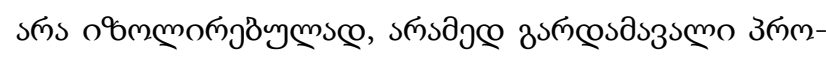

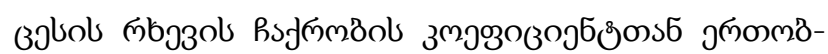

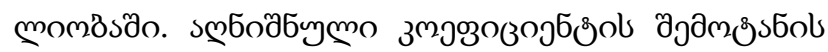

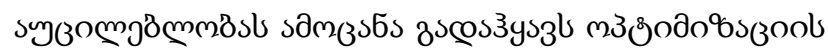

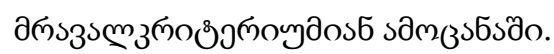

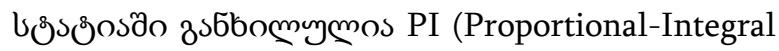

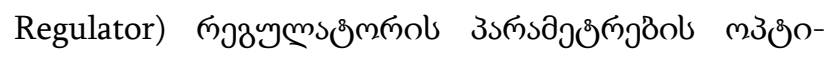

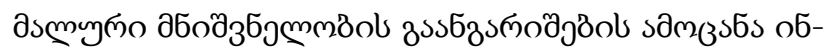

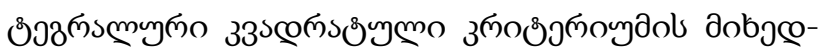

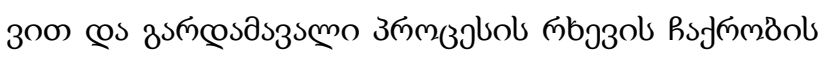

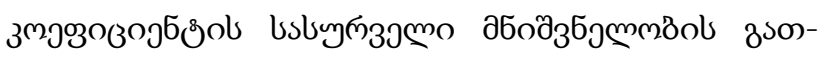

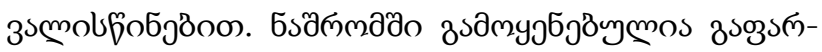

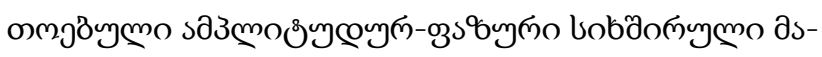

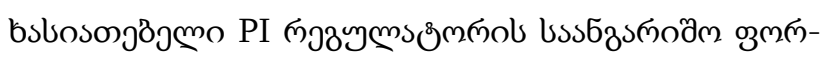

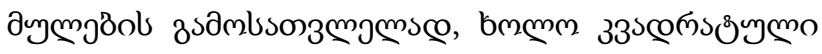

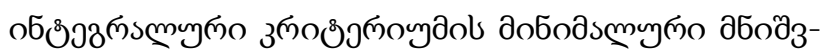

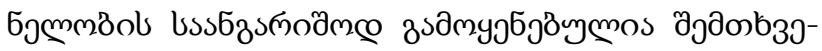

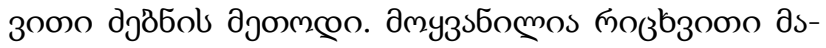

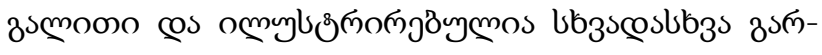

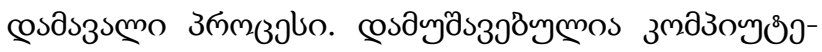

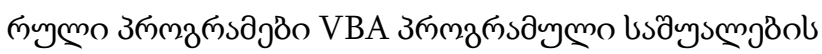

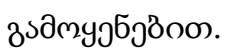




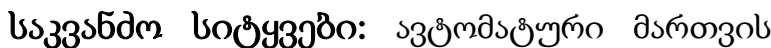

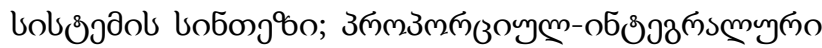

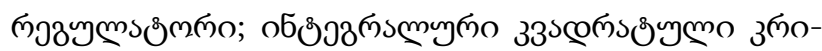

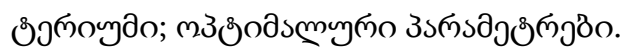

\section{gjuszsmo}

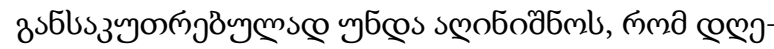

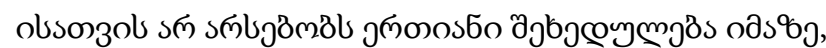

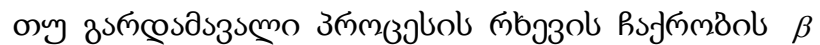

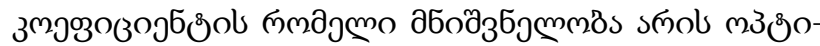

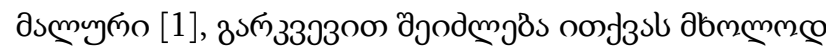

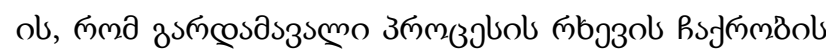

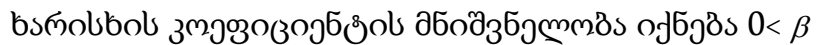

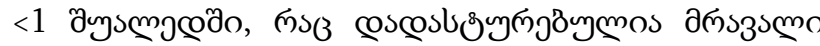

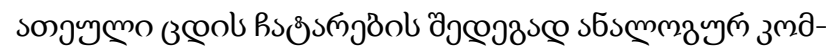

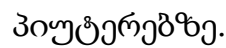

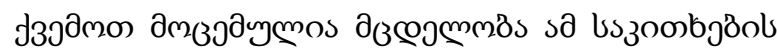

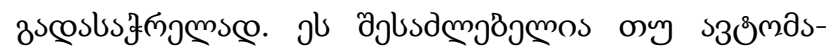

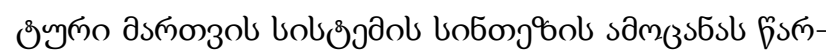

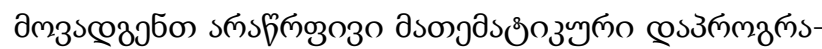

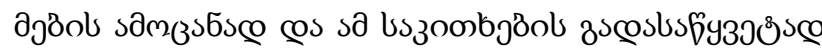

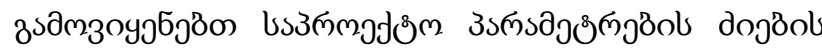

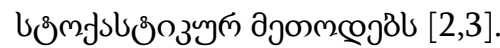

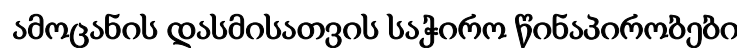

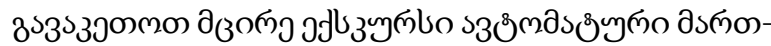

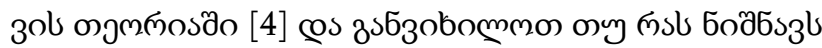

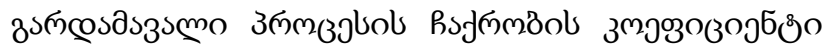

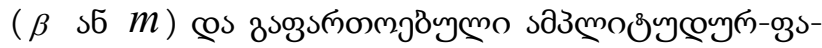

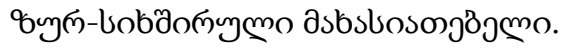

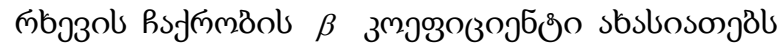

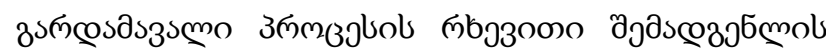

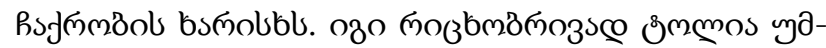

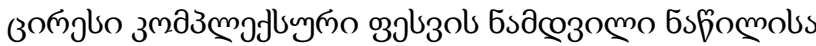

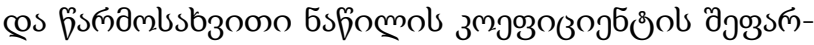

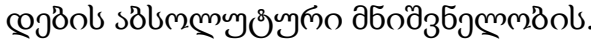

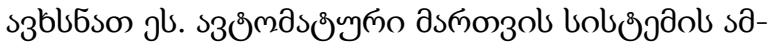

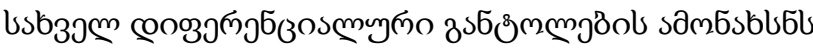
sjablusbg:

$$
y(t)=\sum_{k=1}^{n} A_{k} e^{p_{k} t} .
$$

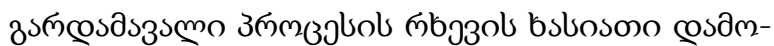

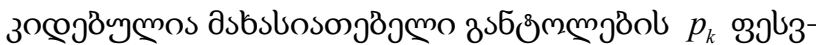

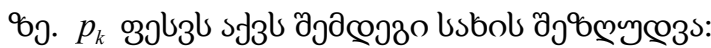

$$
p_{k}=-m \omega+i \omega,
$$

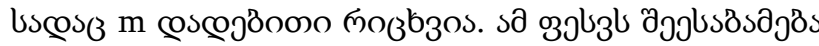

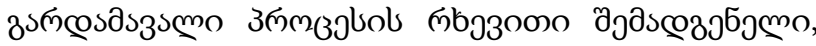
пмалgmbso sjal lsba:

$$
y_{k}(t)=A_{k} e^{-m \omega t} \cos (\omega t),
$$

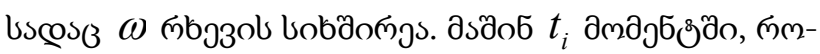

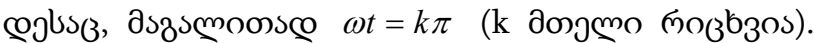

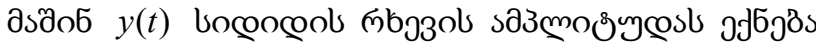

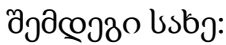

$$
A_{i}=A_{k} e^{-m k \pi},
$$

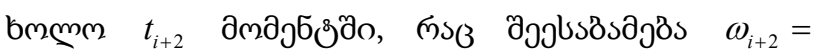

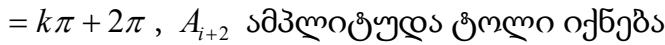

$$
A_{i+2}=A_{k} e^{-m(k \pi+2 \pi)} .
$$

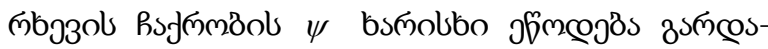

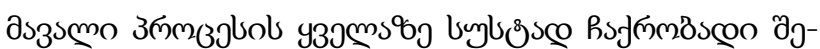

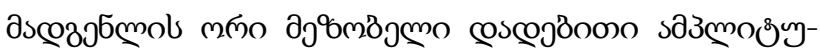

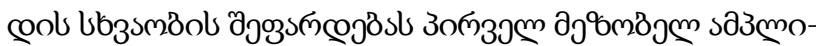

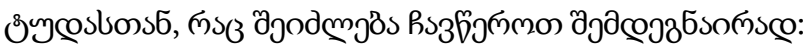

$$
\beta=\frac{\left(\sigma_{\max }\right)_{i}-\left(\sigma_{\max }\right)_{i+2}}{\left(\sigma_{\max }\right)_{i}}=1-\frac{\left(\sigma_{\max }\right)_{i+2}}{\left(\sigma_{\max }\right)_{i}} .
$$




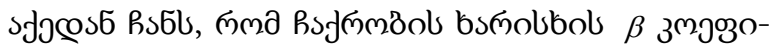

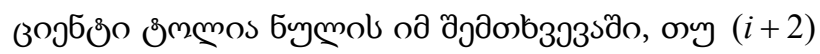

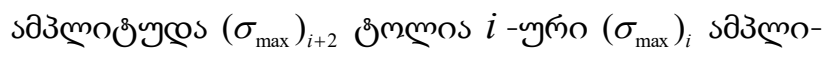

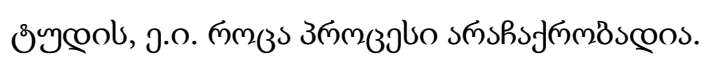

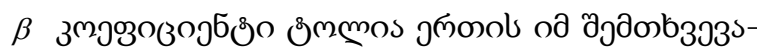

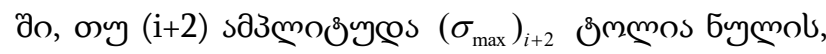

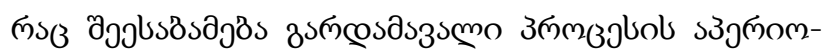
œумॅ озмळаsl.

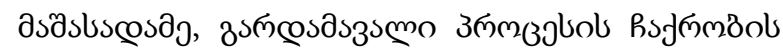

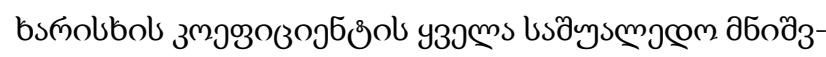

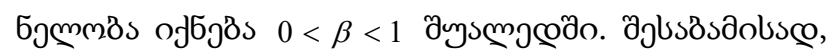

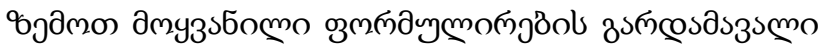

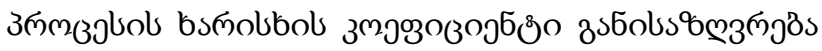
әлдсовбопьм⿻:

$$
\beta=1-\frac{A_{i+2}}{A_{i}}=1-\frac{A_{k} e^{-m(k \pi+2 \pi)}}{A_{k} e^{-m k \pi}}=1-e^{-2 \pi m},
$$

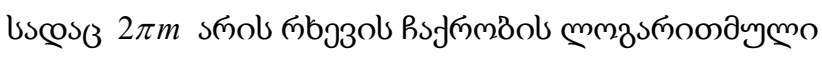

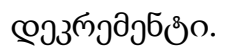

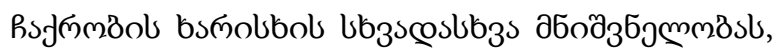

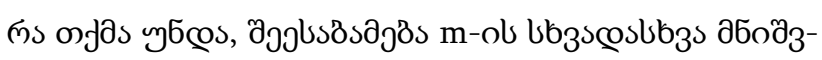

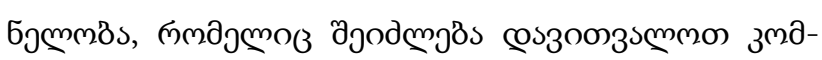

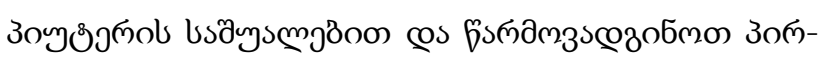

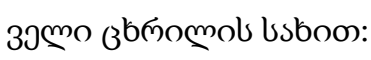

उbஙоmo 1

\begin{tabular}{|c|c|c|c|c|c|c|c|}
\hline$\beta$ & 0.000 & 0.300 & 0.450 & 0.600 & 0.700 & 0.900 & 1.000 \\
\hline$m$ & 0.000 & 0.057 & 0.095 & 0.145 & 0.221 & 0.366 & $\infty$ \\
\hline
\end{tabular}

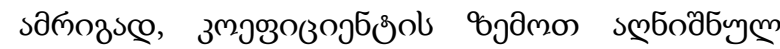

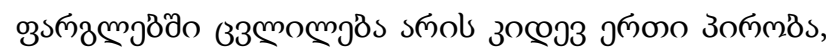

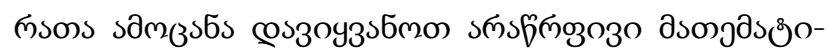

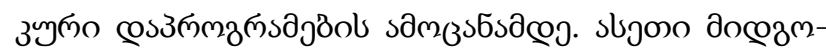

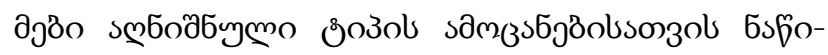

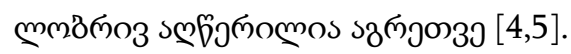

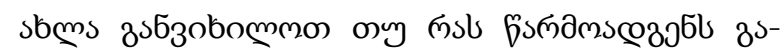

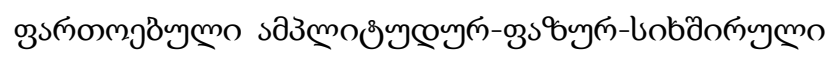
asbsluosong̊̆mo.

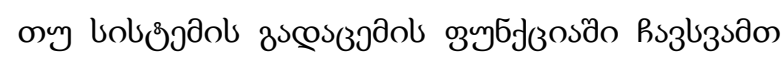

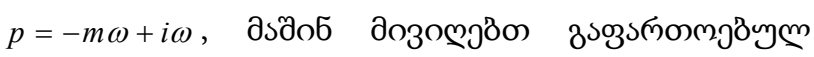

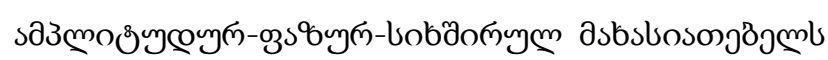

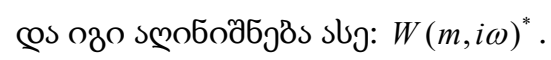

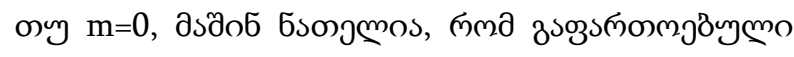

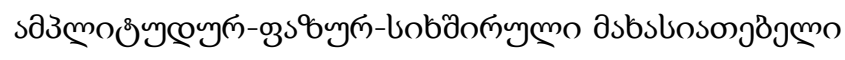

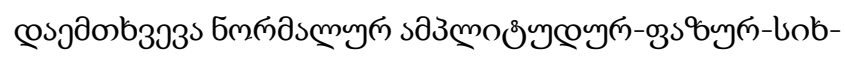

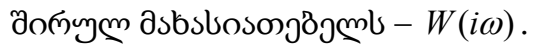

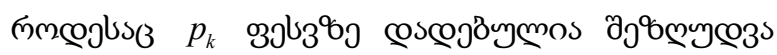

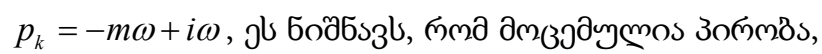

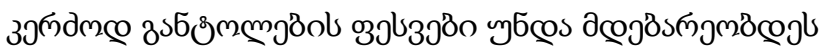

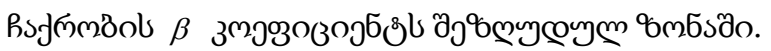

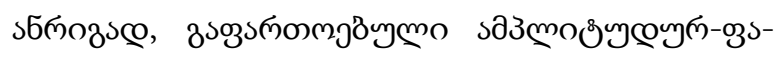

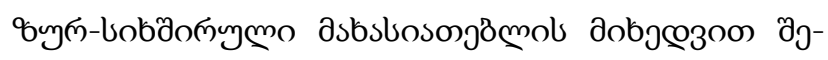

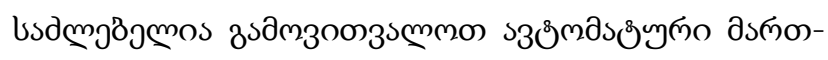

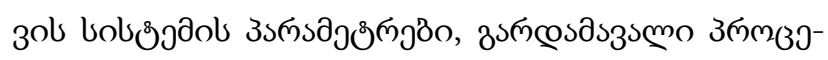

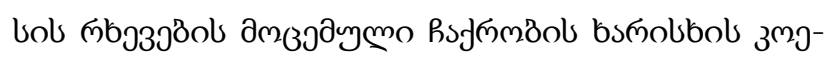

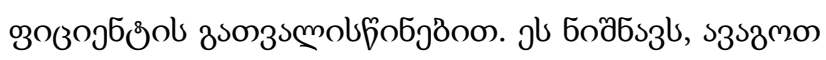

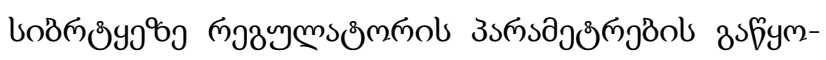

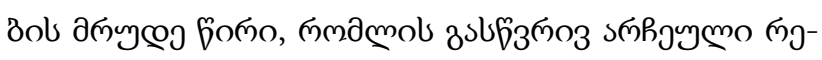

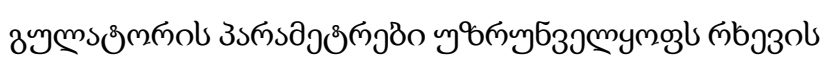

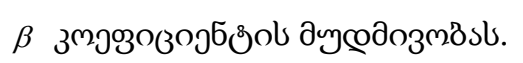




\section{dornoosceo 6sfomo}

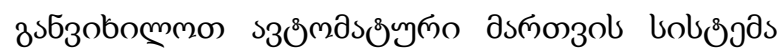

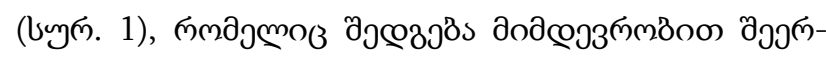

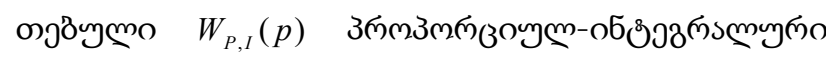

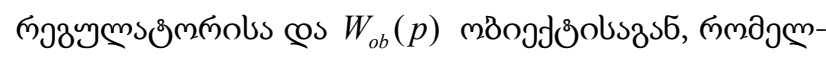

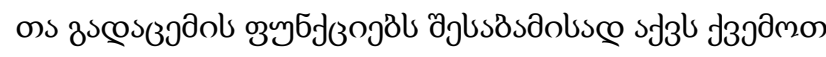
дмyзsбоме bsby:

$$
\begin{gathered}
W_{P, I}=\frac{\left(q_{0}+q_{1} p\right) k}{p}, \\
W_{o b}(p)=\frac{k}{b_{2} p^{2}+b_{1} p+b_{0}},
\end{gathered}
$$

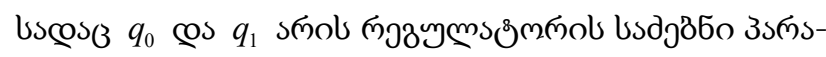

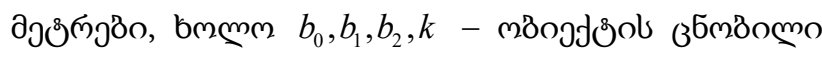

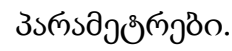

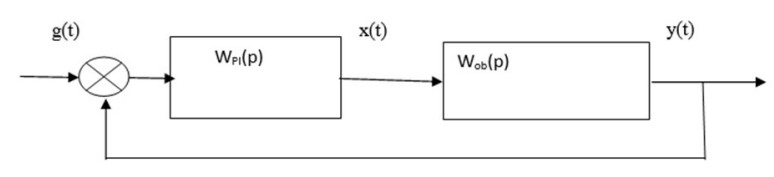

เทตต. 1

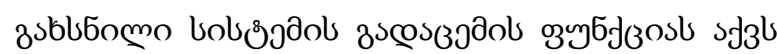
usbo:

$$
\begin{gathered}
W_{o p}(p)=W_{P i}(p) \cdot W_{o b}(p)= \\
=\frac{\left(q_{0}+q_{1} p\right) k}{p\left(b_{2} p^{2}+b_{1} p+b_{0}\right)} .
\end{gathered}
$$

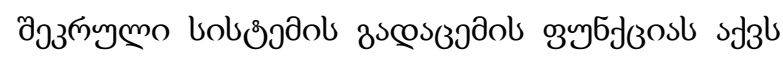
usbo:

$$
\begin{gathered}
W_{c l}(p)=\frac{W_{o p}(p)}{1+W_{o p}(p)}= \\
=\frac{\left(q_{0}+q_{1} p\right) k}{p\left(b_{2} p^{2}+b_{1} p+b_{0}\right)+\left(q_{0}+q_{1} p\right) k} .
\end{gathered}
$$

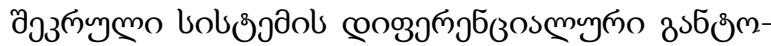

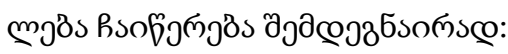

$$
\begin{gathered}
b_{2} y(t)^{\prime \prime \prime}+b_{1} y(t)^{\prime \prime}+\left(b_{0}+q_{1} k\right) y(t)^{\prime}+k q_{0} y(t)= \\
=k q_{0} g(t)+k q g(t)^{\prime},
\end{gathered}
$$

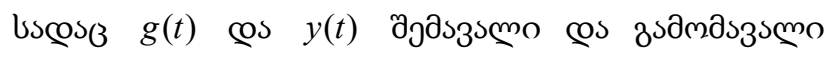

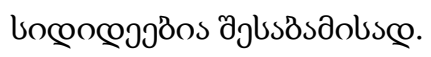

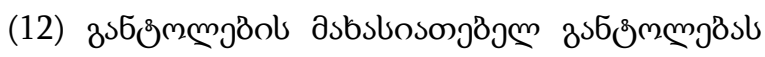
sd3b bsbg:

$$
b_{2} p^{3}+b_{1} p^{2}+\left(b_{0}+q_{1} k\right) p+k q_{0}=0 .
$$

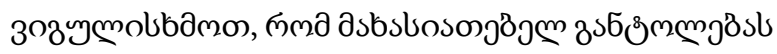

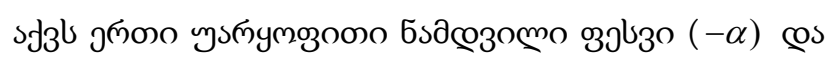

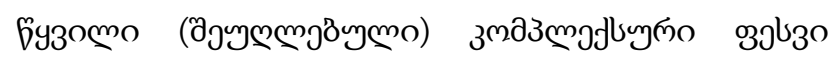

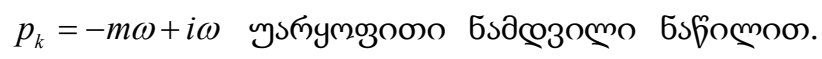

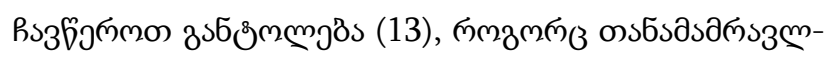

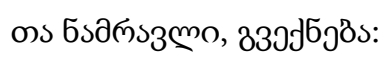

$$
\begin{gathered}
b_{2} p^{3}+b_{1} p^{2}+\left(b_{0}+q_{1} k\right) p+k q_{0}= \\
=b_{2}(p+\alpha)(p+m \omega+i \omega)(p+m \omega-i \omega) .
\end{gathered}
$$

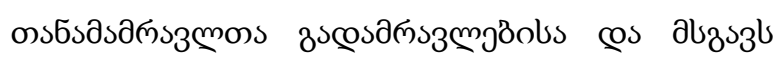

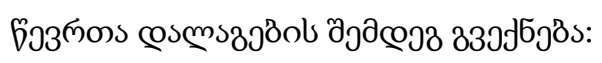

$$
\begin{aligned}
& b_{2} p^{3}+b_{1} p^{2}+\left(b_{0}+q_{1} k\right) p+k q_{0}= \\
& =b_{2}(p+\alpha)(p+m \omega+i \omega)(p+m \omega-i \omega)= \\
& =b_{2} p^{3}+b_{2}(2 m \omega+\alpha) p^{2}+ \\
& +b_{2}\left(m^{2} \omega^{2}+2 m \omega \alpha+\omega^{2}\right) p+ \\
& +b_{2}\left(\alpha m^{2} \omega^{2}+\omega^{2} \alpha\right) .
\end{aligned}
$$

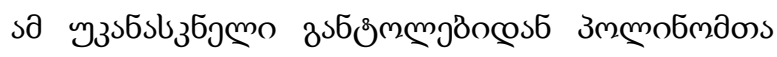

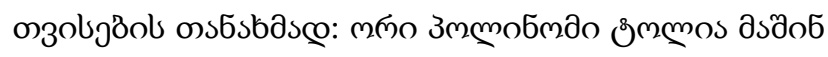

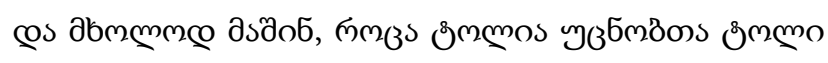

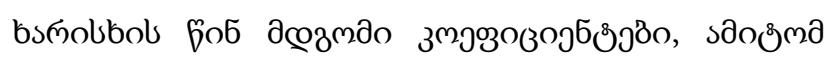

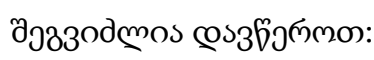




$$
\begin{aligned}
& b_{1}=b_{2}(2 m \omega+\alpha) \\
& b_{0}+k q_{1}=b_{2}\left(m^{2} \omega^{2}+2 m \omega \alpha+\omega^{2}\right) . \\
& k q_{0}=b_{2}\left(\alpha m^{2} \omega^{2}+\omega^{2} \alpha\right) .
\end{aligned}
$$

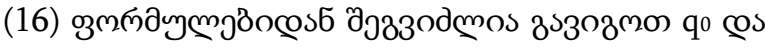

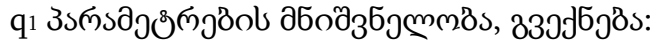

$$
\begin{aligned}
& \alpha=\frac{b_{1}}{b_{2}}-2 m \omega \\
& q_{0}=\frac{1}{k}\left(m^{2}+1\right)\left(b_{1}-2 b_{2} m \omega\right) \omega^{2} \\
& q_{1}=\frac{1}{k}\left(b_{2} \omega^{2}+2 m b_{1} \omega-3 b_{2} m^{2} \omega^{2}-b_{0}\right) .
\end{aligned}
$$

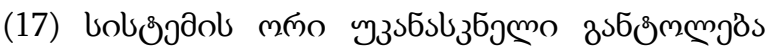

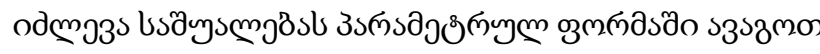

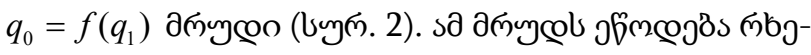

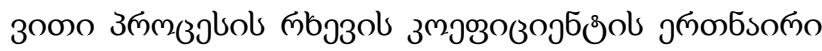

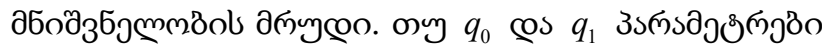

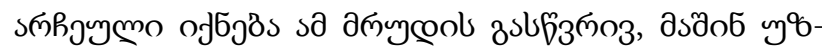

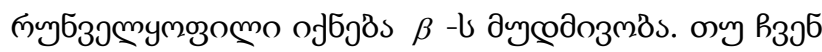

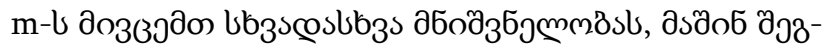

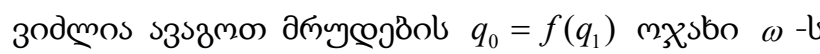

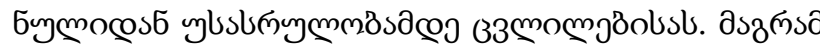

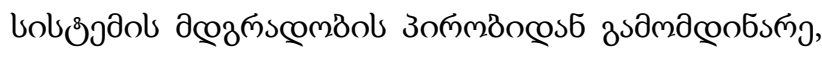

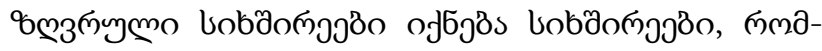

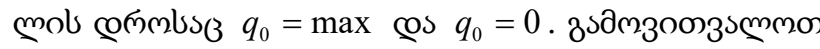

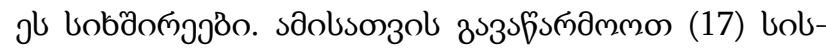

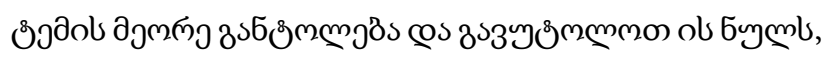

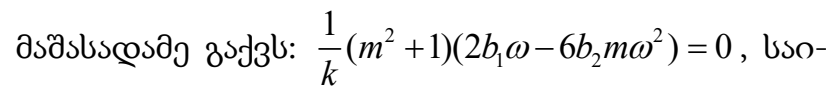
cos6so: $\omega=\frac{b_{1}}{3 b_{2} m}$.

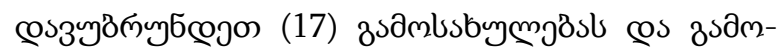

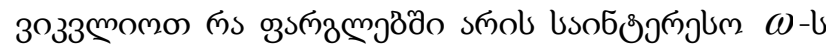

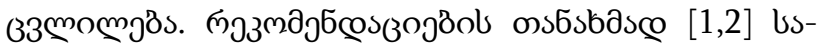

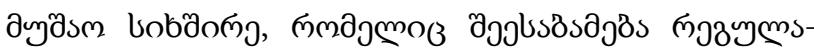

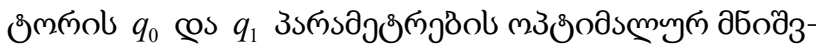

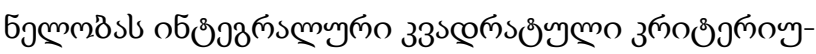

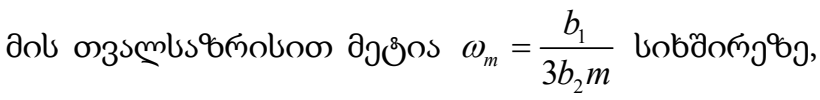

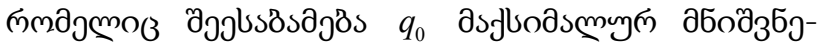

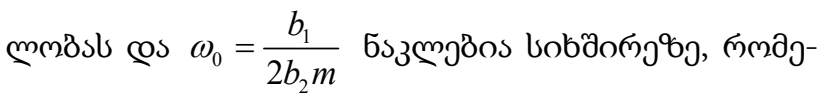

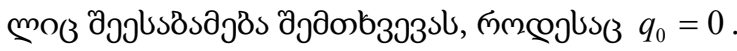

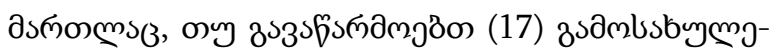

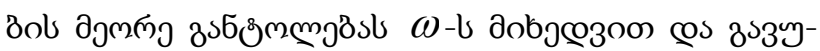

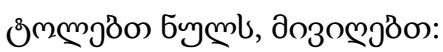

$$
\begin{aligned}
& {\left[\frac{1}{k}\left(m^{2}+1\right)\left(b_{1} \omega^{2}-2 b_{2} \omega^{3} m\right)\right]^{\prime}=} \\
& \left.=2 b_{1} \omega-6 b_{2} \omega^{2}\right)=\omega\left(2 b_{1}-3 b_{2} m \omega\right)=0 .
\end{aligned}
$$

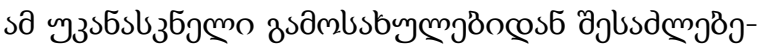

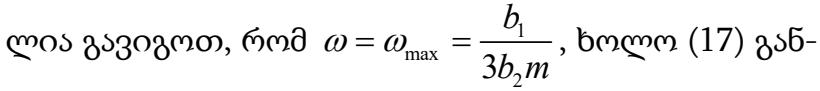

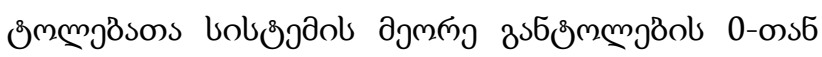

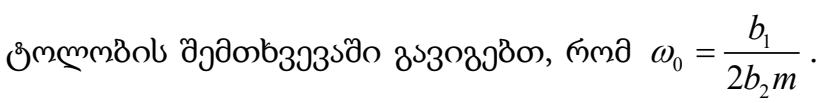

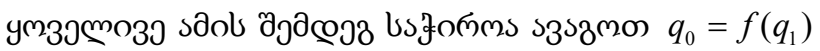

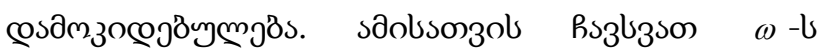

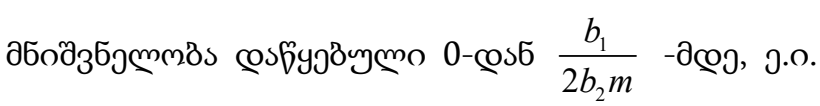
วsธงว Łsธsว $q_{0}=0$.

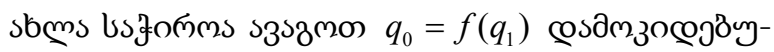

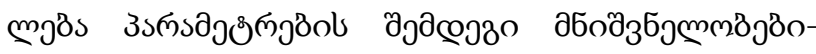

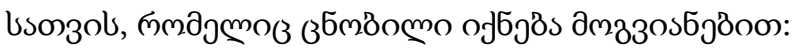




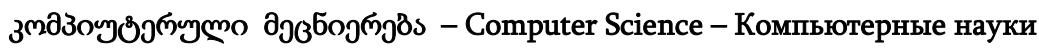

$I_{0 \min }^{(\min )}=8.40432 E(-05), m=0.205,(\beta=0.724013), \omega=23.636558, q_{0}=0.719565, q_{1}=2.300645 ;$

$I_{0 \min }^{(\text {Average })}=0.010936472, m=0.22,(\beta=0.748824), \omega=21.8877, q_{0}=0.7421, q_{1}=1.983277$;

$I_{0 \min }^{(\max )}=0.013782417, m=0.335,(\beta=0.878008), \omega=13.78847, q_{0}=0.644297, q_{1}=0.833981$.

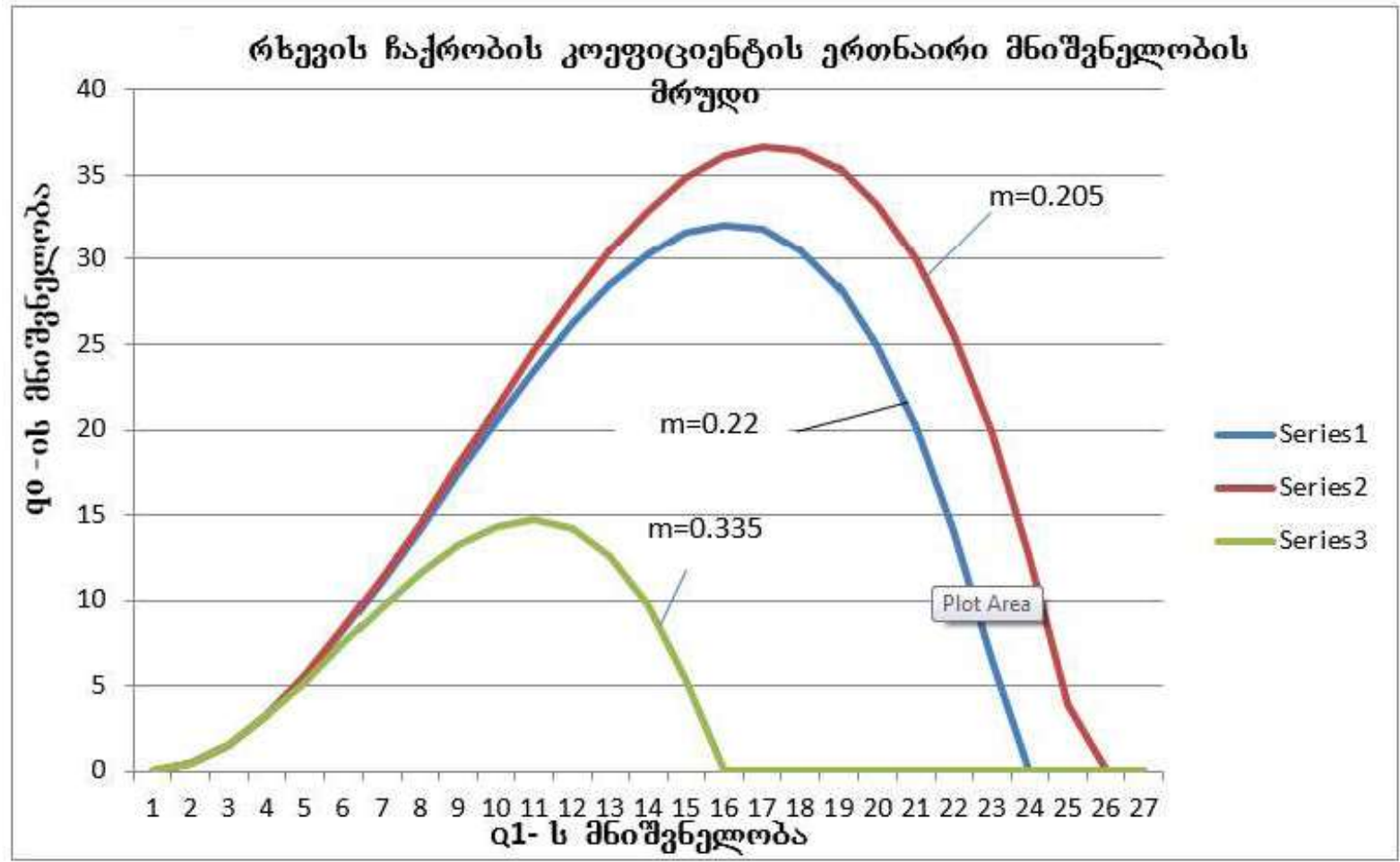

เуy๓. 2

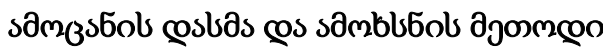

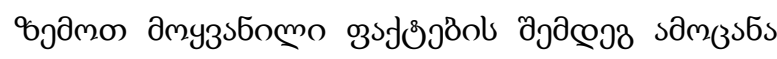

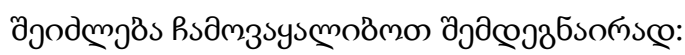

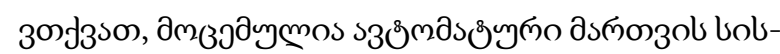

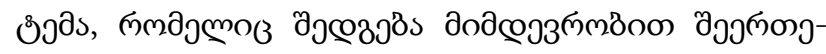

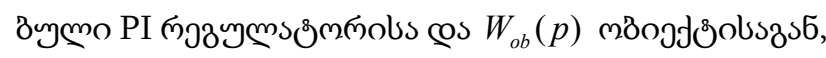

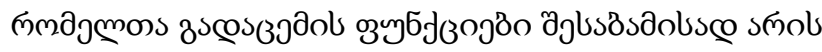

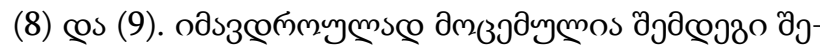
६муч⿻3วð̊:

$$
\begin{aligned}
& \frac{b_{1}}{3 b_{2} m} \leq \omega \leq \frac{b_{1}}{2 b_{2} m}, \\
& \alpha=\frac{b_{1}}{b_{2}}-2 m \omega, \\
& q_{0}=\frac{1}{k}\left(m^{2}+1\right)\left(b_{1}-2 b_{2} m \omega\right) \omega^{2} \geq 0, \\
& q_{1}=\frac{1}{k}\left(b_{2} \omega^{2}+2 m b_{1} \omega-3 b_{2} m^{2} \omega^{2}-b_{0}\right) \geq 0, \\
& q_{0}=f\left(q_{1}\right) .
\end{aligned}
$$

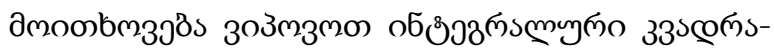

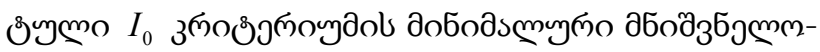

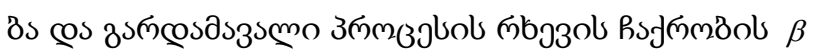

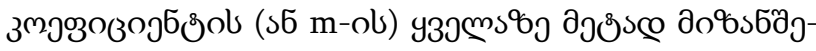




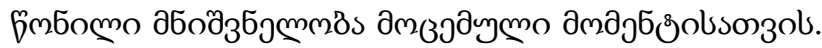

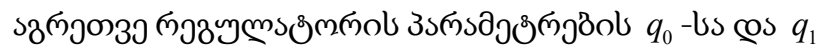

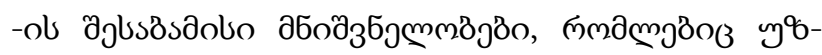

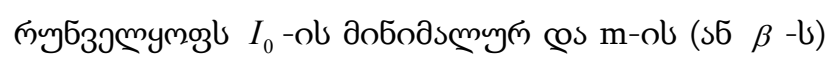

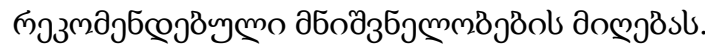

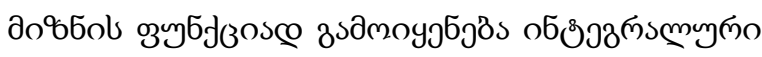

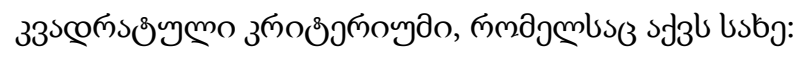

$$
I_{0}=\int_{0}^{\infty} y^{2}(t) d t=\frac{1}{\pi} \int_{0}^{\infty}\left|Y_{m, n}(i \omega)\right|^{2} d \omega
$$

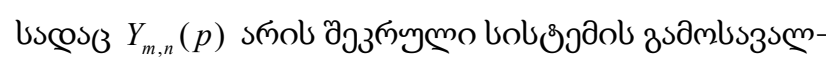

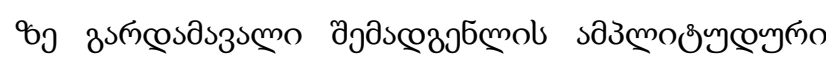

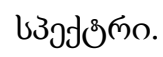

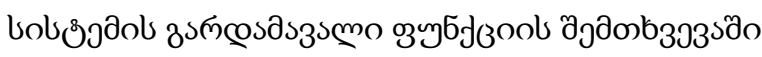

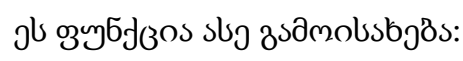

$$
y(t)=y_{s t}(t)-y(t),
$$

bmen $\quad Y_{m, n}(p)=\frac{W_{c l}(0)-W_{c l}(p)}{p}$,

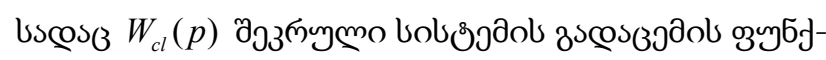

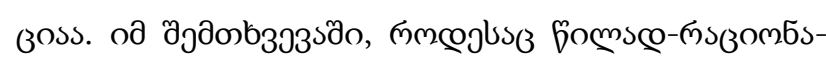

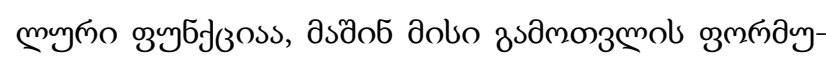

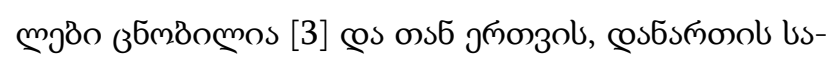

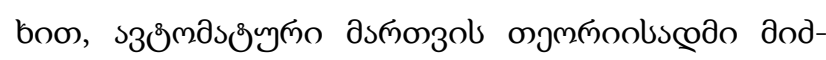

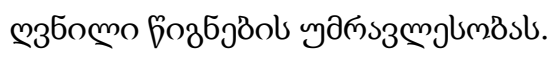

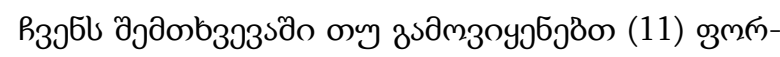

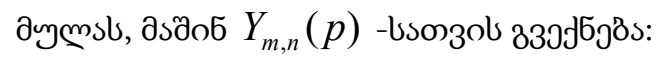

$$
\begin{aligned}
& Y_{m, n}(p)=\frac{1}{p}\left[W_{c l}(0)-W_{c l}(p)\right]= \\
& =\frac{1}{p}\left[1-\frac{\left(q_{0}+q_{1} p\right) k}{p\left(b_{2} p^{2}+b_{1} p+b_{0)}+\left(q_{0}+q_{1} p\right) k\right.}=\right. \\
& =\frac{b_{2} p^{2}+b_{1} p+b_{0}}{b_{2} p^{3}+b_{1} p^{2}+\left(b_{0}+q_{1} k\right) p+q_{0} k} .
\end{aligned}
$$

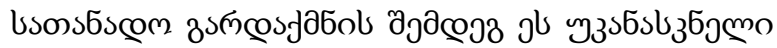

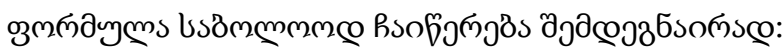

$$
\begin{aligned}
Y_{m, n}(p)= & \frac{b p^{2}+b_{1} p+b_{0}}{c_{3} p^{3}+c_{2} p^{2}+c_{1} p+c_{0}}, \\
& c_{3}=b_{2}, \\
& c_{2}=b_{1}, \\
& c_{1}=b_{0}+q_{1} k, \\
& c_{0}=q_{0} k .
\end{aligned}
$$

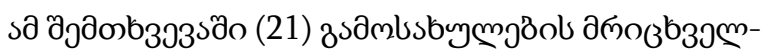

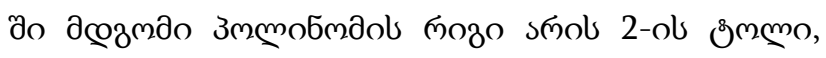

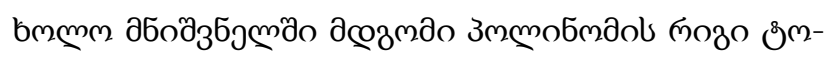

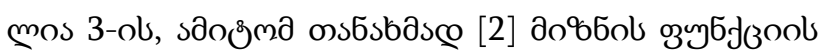

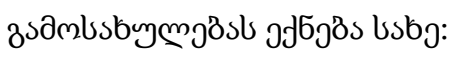

$$
I_{0}=\frac{b_{2}^{2} c_{0} c_{1}+\left(b_{1}^{2}-2 b_{0} b_{2}\right) c_{0} c_{3}+b_{0}^{2} c_{2} c_{3}}{2 c_{0} c_{3}\left(-c_{0} c_{3}+c_{1} c_{2}\right)} .
$$

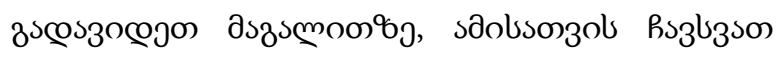

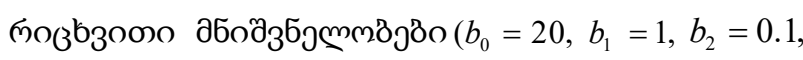

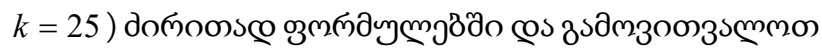

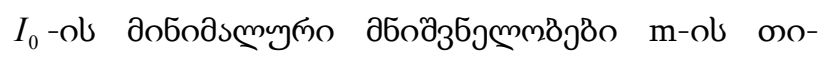

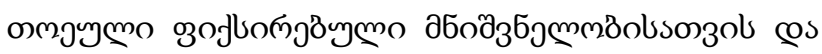

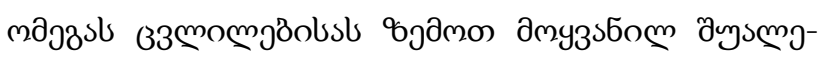

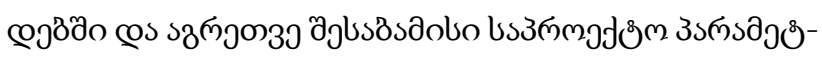

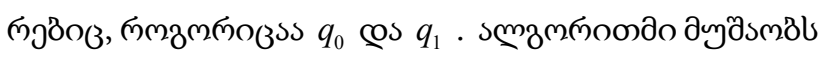

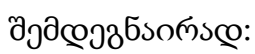

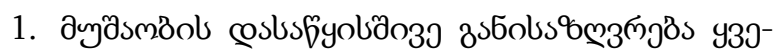

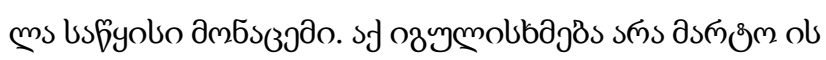

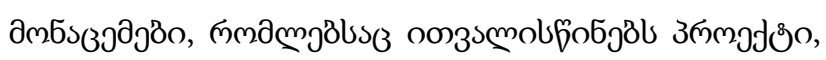

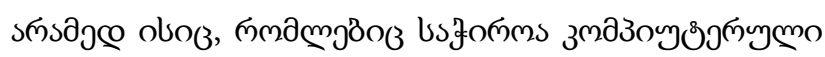

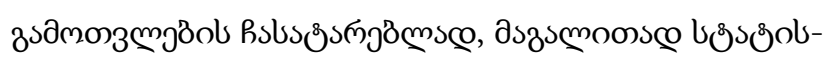

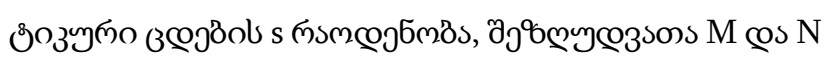

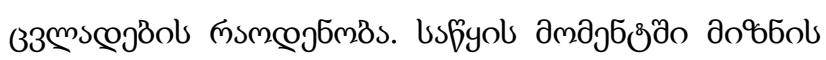




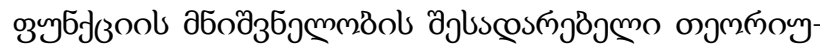

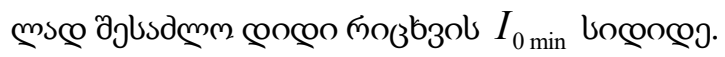

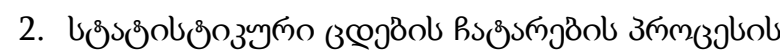

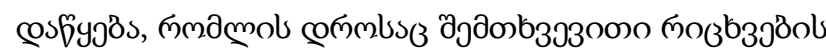

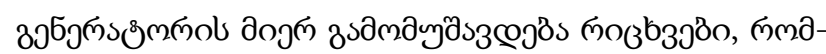

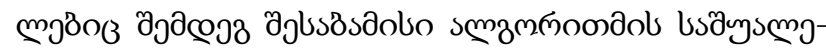

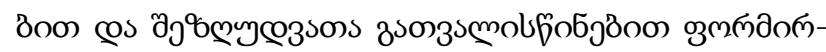

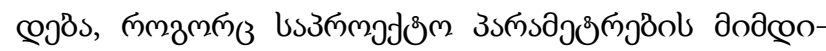

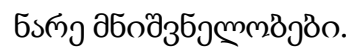

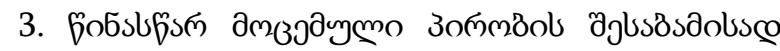

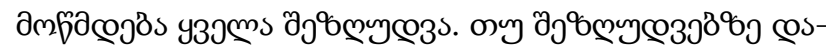

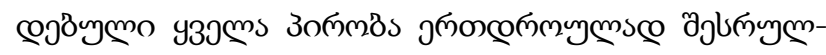

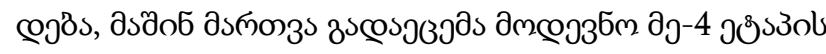

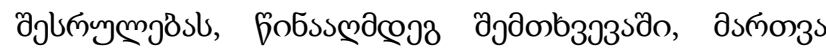

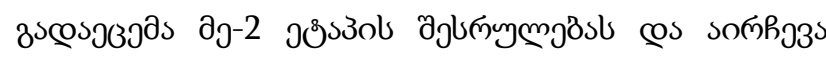

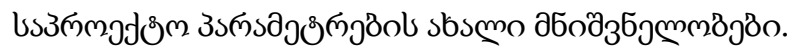

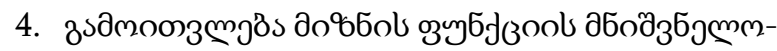

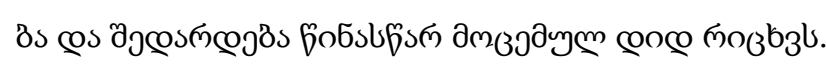

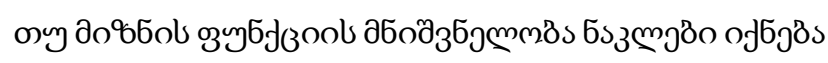

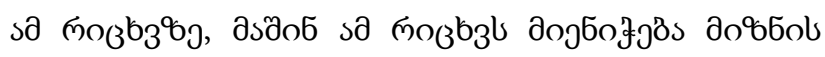

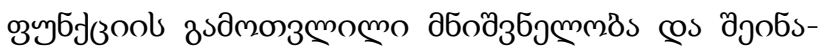

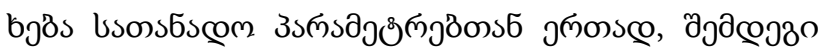

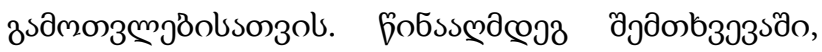

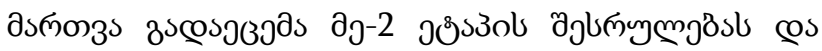

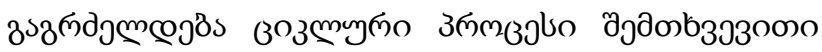

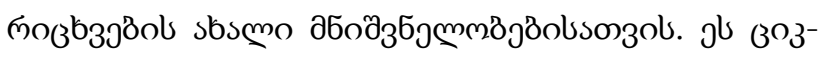

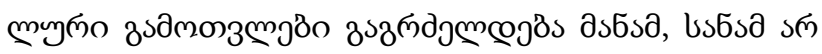

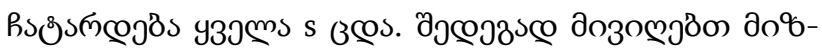
bol oзy5

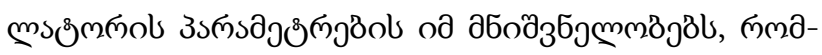

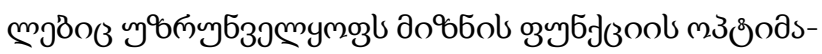

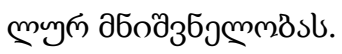

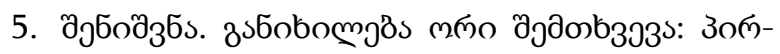

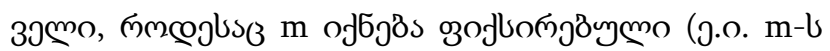

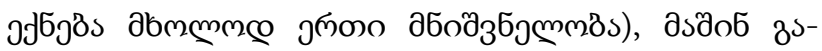

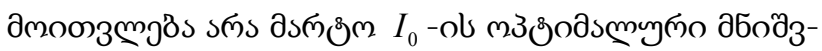

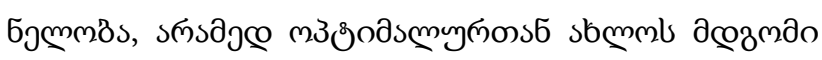

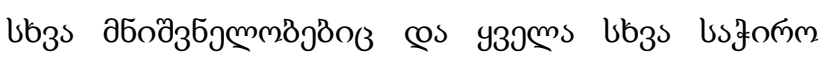

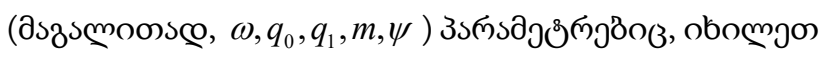
aృ-2 зйомо.

3bங̆омо 2

\begin{tabular}{|c|l|l|l|l|l|}
\hline$I_{0}$ & \multicolumn{1}{|c|}{$\omega$} & \multicolumn{1}{|c|}{$q_{0}$} & \multicolumn{1}{|c|}{$q_{1}$} & \multicolumn{1}{|c|}{$m$} & $\beta$ \\
\hline $7.584121789 \mathrm{E}-02$ & 13.460435341 & 0.7911609485 & 0.801473368 & 0.335 & 0.761598726 \\
\hline $9.892724455 \mathrm{E}-03$ & 13.804677069 & 0.6366017043 & 0.835602362 & 0.335 & 0.761598726 \\
\hline $3.330686415 \mathrm{E}-03$ & 13.856777163 & 0.6115971389 & 0.840822486 & 0.335 & 0.761598726 \\
\hline $1.734377745 \mathrm{E}-03$ & 13.850722695 & 0.6145247229 & 0.840215124 & 0.335 & 0.761598726 \\
\hline $6.696982415 \mathrm{E}-04$ & 13.841486051 & 0.6189804412 & 0.839288913 & 0.335 & 0.761598726 \\
\hline
\end{tabular}

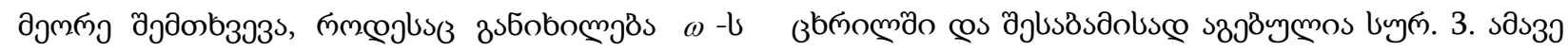

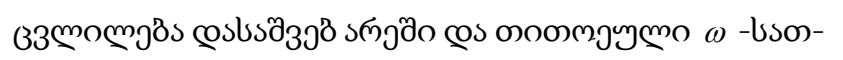

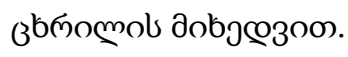

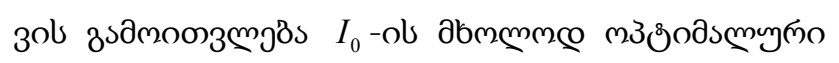

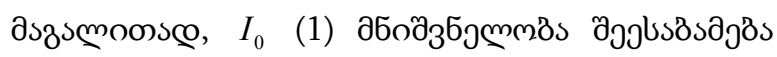

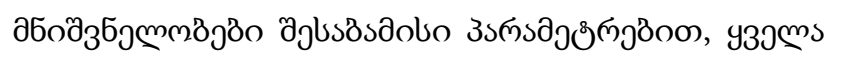

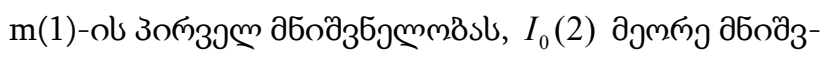

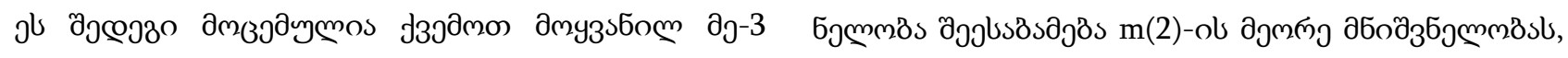




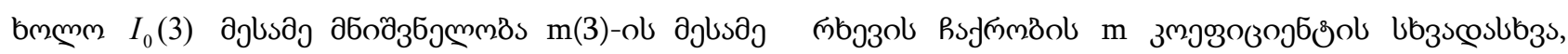

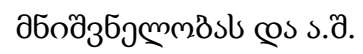

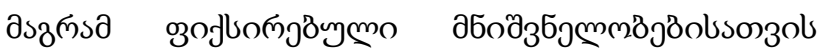

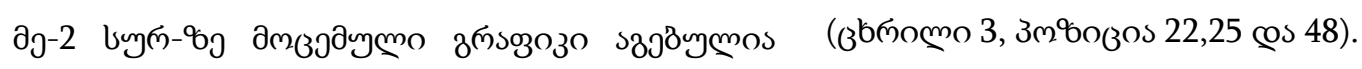

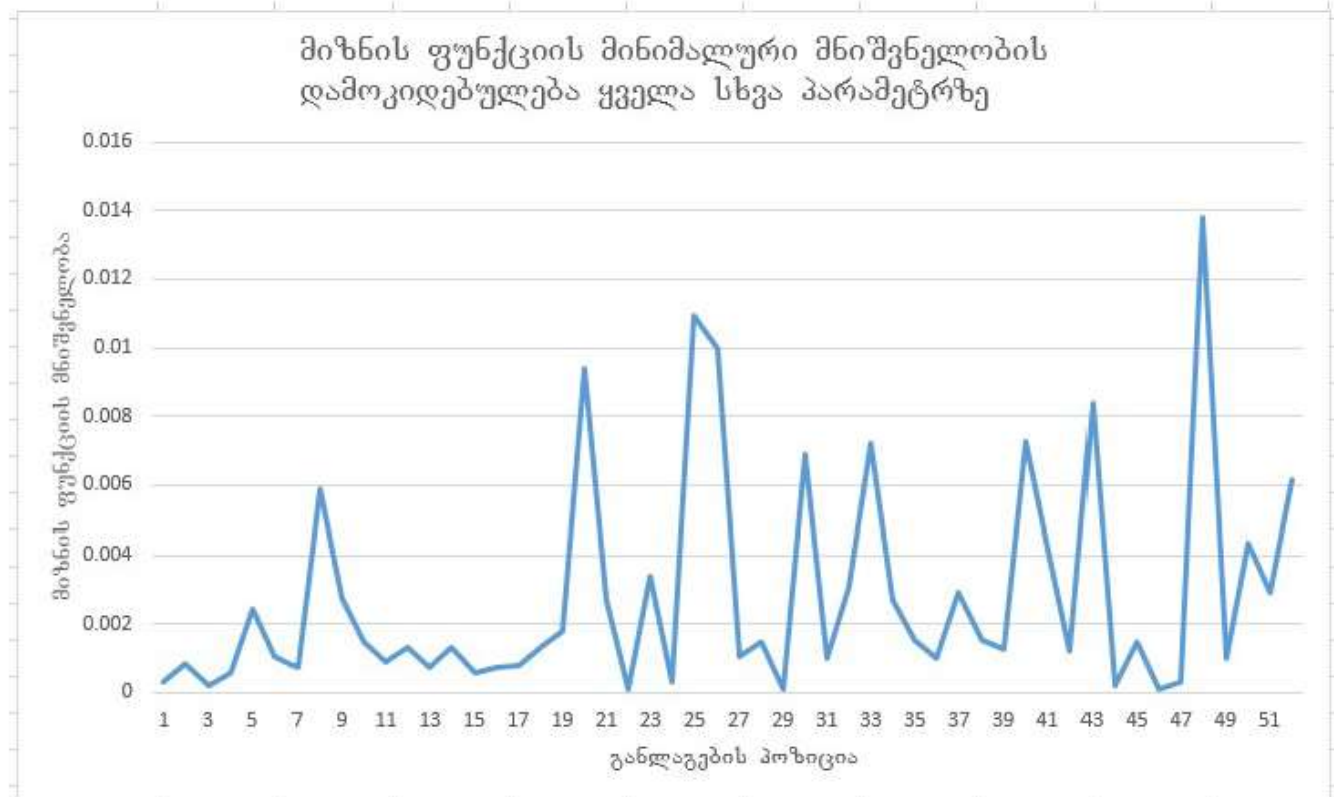

เugn. 3

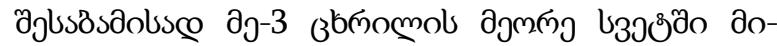

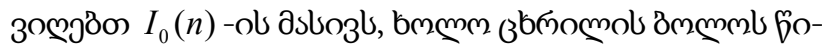

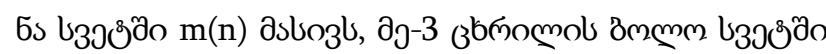

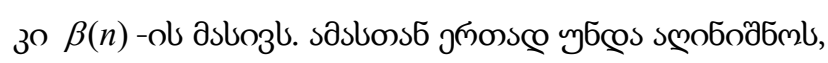

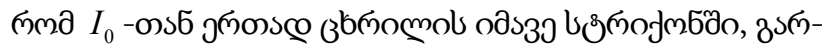

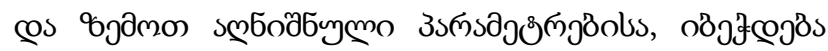

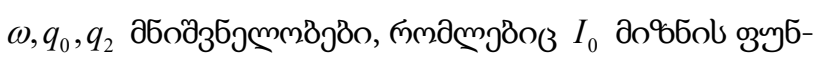

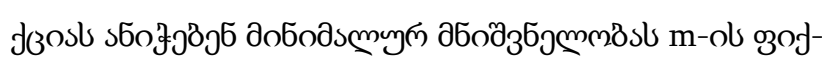

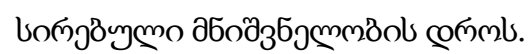

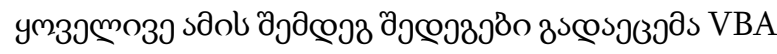

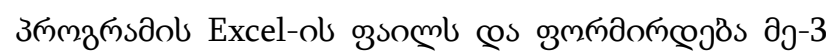

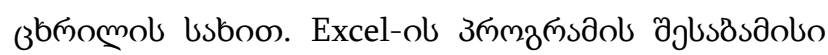

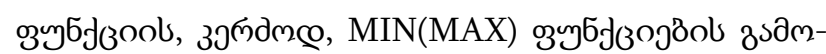

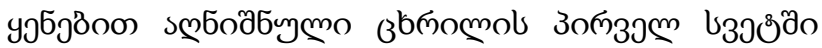

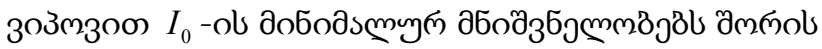

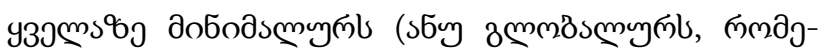

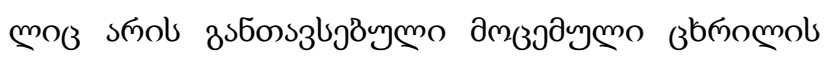

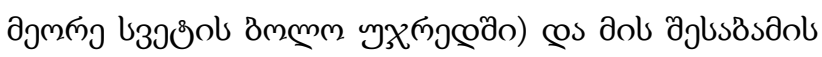

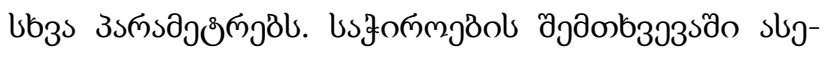

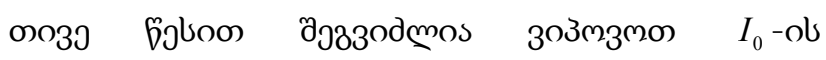

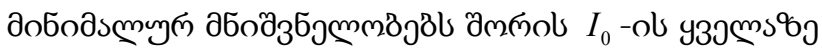

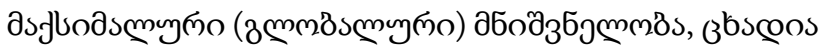

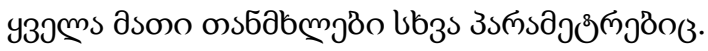

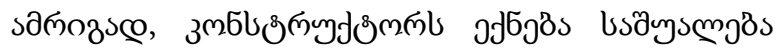

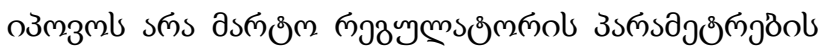

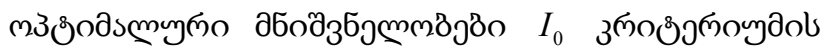




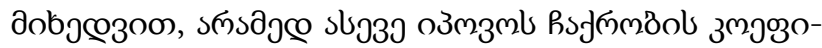

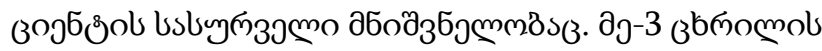

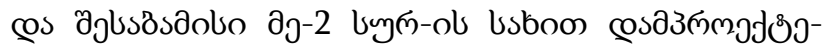

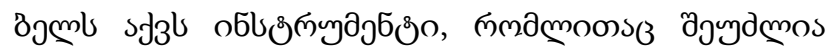

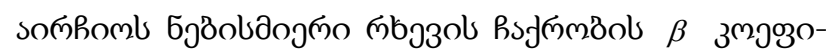

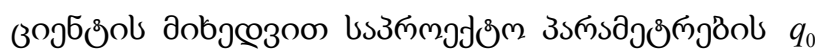

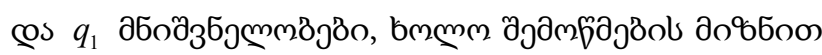

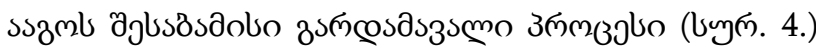

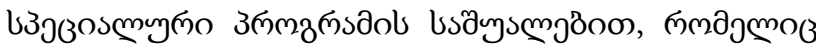

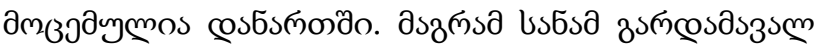

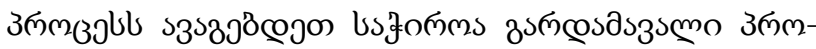

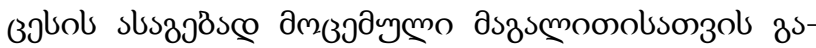

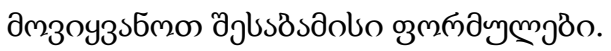

3bங̆mo 3

\begin{tabular}{|c|c|c|c|c|c|c|}
\hline №№ & $I_{0}$ & $\omega$ & $q_{0}$ & $q_{1}$ & $m$ & $\beta$ \\
\hline 1 & 0.000327069 & 48.11 & 3.534627 & 9.32542 & 0.1 & 0.466342 \\
\hline 2 & 0.000834774 & 46.17784 & 2.609959 & 8.595352 & 0.105 & 0.482838 \\
\hline 3 & 0.00022522 & 44.32501 & 1.976537 & 7.923609 & 0.11 & 0.498825 \\
\hline 4 & 0.000567988 & 42.48404 & 1.672732 & 7.283993 & 0.115 & 0.514317 \\
\hline 5 & 0.002418327 & 40.7114 & 1.541836 & 6.694099 & 0.12 & 0.529331 \\
\hline 6 & 0.00104409 & 39.15429 & 1.316784 & 6.196328 & 0.125 & 0.54388 \\
\hline 7 & 0.000729015 & 37.66684 & 1.192422 & 5.739169 & 0.13 & 0.55798 \\
\hline 8 & 0.005882586 & 36.3661 & 0.97576 & 5.353497 & 0.135 & 0.571644 \\
\hline 9 & 0.002769024 & 34.94147 & 1.077471 & 4.947813 & 0.14 & 0.584885 \\
\hline 10 & 0.001472994 & 33.74409 & 0.996187 & 4.6188 & 0.145 & 0.597717 \\
\hline 11 & 0.000923049 & 32.63047 & 0.918246 & 4.323075 & 0.15 & 0.610153 \\
\hline 12 & 0.001344304 & 31.53717 & 0.910442 & 4.042691 & 0.155 & 0.622204 \\
\hline 13 & 0.000727617 & 30.55056 & 0.856991 & 3.797673 & 0.16 & 0.633882 \\
\hline 14 & 0.001300217 & 29.58625 & 0.850755 & 3.565948 & 0.165 & 0.6452 \\
\hline 15 & 0.000575964 & 28.69928 & 0.821169 & 3.359263 & 0.17 & 0.656167 \\
\hline 16 & 0.000716428 & 27.85467 & 0.802414 & 3.168359 & 0.175 & 0.666796 \\
\hline 17 & 0.00080005 & 27.06673 & 0.774431 & 2.995354 & 0.18 & 0.677096 \\
\hline 18 & 0.001346505 & 26.29614 & 0.773586 & 2.831138 & 0.185 & 0.687078 \\
\hline 19 & 0.001805518 & 25.59772 & 0.740998 & 2.686206 & 0.19 & 0.696751 \\
\hline 20 & 0.009417903 & 24.84586 & 0.794875 & 2.535181 & 0.195 & 0.706125 \\
\hline 21 & 0.002707012 & 24.24008 & 0.742996 & 2.416129 & 0.2 & 0.715209 \\
\hline 22 & $8.40432 \mathrm{E}-05$ & 23.63658 & 0.719565 & 2.300645 & 0.205 & 0.724013 \\
\hline
\end{tabular}




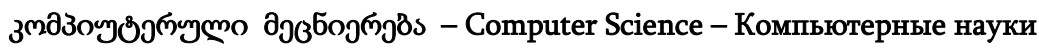

\begin{tabular}{|c|c|c|c|c|c|c|}
\hline & & & & & & 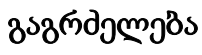 \\
\hline 23 & 0.003377463 & 23.0643 & 0.695371 & 2.193814 & 0.21 & 0.732544 \\
\hline 24 & 0.000301596 & 22.48215 & 0.703692 & 2.08811 & 0.215 & 0.740812 \\
\hline 25 & 0.010936472 & 21.88777 & 0.7421 & 1.983277 & 0.22 & 0.748824 \\
\hline 26 & 0.010002039 & 21.4748 & 0.651849 & 1.911055 & 0.225 & 0.756588 \\
\hline 27 & 0.001041293 & 20.94042 & 0.678525 & 1.820948 & 0.23 & 0.764112 \\
\hline 28 & 0.001486104 & 20.46865 & 0.671524 & 1.743025 & 0.235 & 0.771404 \\
\hline 29 & 8.87519E-05 & 20.00764 & 0.67117 & 1.668678 & 0.24 & 0.778471 \\
\hline 30 & 0.006920061 & 19.53897 & 0.689432 & 1.595059 & 0.245 & 0.785319 \\
\hline 31 & 0.000995069 & 19.14599 & 0.665239 & 1.534269 & 0.25 & 0.791955 \\
\hline 32 & 0.00305399 & 18.7588 & 0.649129 & 1.475668 & 0.255 & 0.798386 \\
\hline 33 & 0.00720793 & 18.38583 & 0.634255 & 1.420363 & 0.26 & 0.804618 \\
\hline 34 & 0.002685301 & 17.96988 & 0.657963 & 1.360505 & 0.265 & 0.810658 \\
\hline 35 & 0.001508556 & 17.61272 & 0.651173 & 1.309897 & 0.27 & 0.816511 \\
\hline 36 & 0.000988335 & 17.27325 & 0.641488 & 1.262706 & 0.275 & 0.822183 \\
\hline 37 & 0.002890809 & 16.94291 & 0.633959 & 1.217702 & 0.28 & 0.82768 \\
\hline 38 & 0.001516707 & 16.59876 & 0.641916 & 1.171979 & 0.285 & 0.833006 \\
\hline 39 & 0.001290848 & 16.28373 & 0.63866 & 1.130823 & 0.29 & 0.838169 \\
\hline 40 & 0.007284536 & 16.01083 & 0.617068 & 1.095539 & 0.295 & 0.843171 \\
\hline 41 & 0.004165458 & 15.70269 & 0.621803 & 1.056862 & 0.3 & 0.848019 \\
\hline 42 & 0.001222366 & 15.39467 & 0.631287 & 1.019055 & 0.305 & 0.852717 \\
\hline 43 & 0.008400314 & 15.08735 & 0.644561 & 0.982178 & 0.31 & 0.85727 \\
\hline 44 & 0.000206247 & 14.84976 & 0.625042 & 0.953708 & 0.315 & 0.861682 \\
\hline 45 & 0.001454413 & 14.58244 & 0.625665 & 0.922599 & 0.32 & 0.865958 \\
\hline 46 & 0.000108136 & 14.33265 & 0.621209 & 0.893972 & 0.325 & 0.870101 \\
\hline 47 & 0.000287527 & 14.08628 & 0.618779 & 0.866271 & 0.33 & 0.874117 \\
\hline 48 & 0.013782417 & 13.78847 & 0.644293 & 0.833981 & 0.335 & 0.878008 \\
\hline 49 & 0.001011575 & 13.61335 & 0.614385 & 0.814496 & 0.34 & 0.881779 \\
\hline 50 & 0.004355172 & 13.36397 & 0.622632 & 0.78814 & 0.345 & 0.885434 \\
\hline 51 & 0.002929381 & 13.14708 & 0.618567 & 0.765418 & 0.35 & 0.888975 \\
\hline 52 & 0.006169637 & 12.91724 & 0.622842 & 0.741935 & 0.355 & 0.892407 \\
\hline $\begin{array}{c}\text { Iomin }= \\
=\mathrm{I}_{0 \max }\end{array}$ & 0.013782417 & & & & & \\
\hline Iomin $==$ IoMin & $8.40432 \mathrm{E}-05$ & & & & & \\
\hline
\end{tabular}




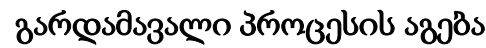

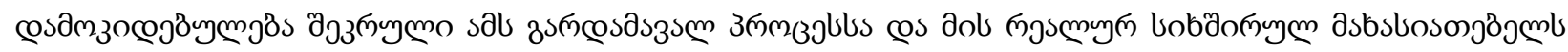

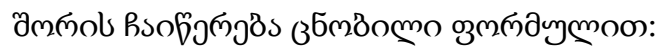

$$
y(t)=\frac{2}{\pi} \int_{0}^{\infty} \frac{U(\omega)}{\omega} \operatorname{Sin}(\omega t) .
$$

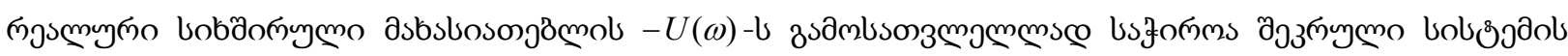

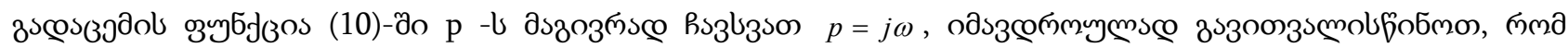

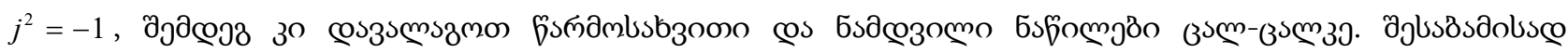
дозомวठัо:

$$
\begin{gathered}
W_{c l}(j \omega)=\frac{\left(q_{0}+j q_{1} \omega\right) k}{j \omega\left(-b_{2} \omega^{2}+j b_{1} \omega+b_{0}\right)+\left(q_{0}+j q_{1} \omega\right) k}=\frac{\left(q_{0}+j q_{1} \omega\right) k}{-j b_{2} \omega^{3}-b_{1} \omega^{2}+j b_{0} \omega+q_{0} k+j q_{1} k \omega}= \\
=\frac{\left(q_{0}+j q_{1} k\right)}{\left.\left(q_{0} k-b_{1} \omega^{2}\right)-j\left[b_{2} \omega^{3}+9 b_{0}+q_{1} k\right) \omega\right]} .
\end{gathered}
$$

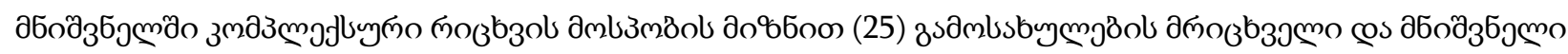

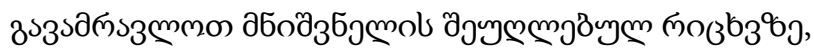

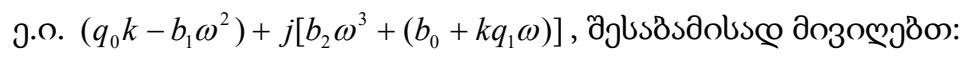

$$
W_{c l}(j \omega)=\frac{\left.k\left(q_{0}+j q_{1} \omega\right)\left\{q_{0} k-b_{1} \omega^{2}\right)+j\left[b_{2} \omega^{3}+\left(b_{0}+k q_{1}\right) \omega\right]\right\}}{\left(q_{0} k-b_{1} \omega^{2}\right)^{2}+\left[b_{2} \omega^{3}+\left(b_{0}+k q_{1} \omega\right]^{2}\right.} .
$$

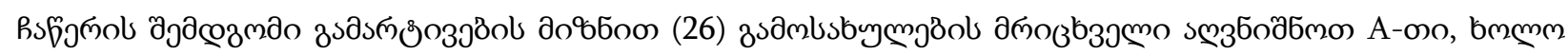
абодзбэмю - В-оо.

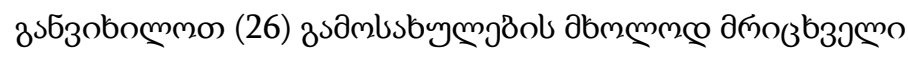

$$
A=k\left\{q_{0}\left(q_{0} k-b_{1} \omega^{2}\right)+j q_{0}\left[b_{2} \omega^{3}+\left(b_{0}+k q_{1}\right) \omega\right]+j q_{1} \omega\left(q_{0} k-b_{1} \omega^{2}\right)-q_{1} \omega\left[b_{2} \omega^{3}+\left(b_{0}+k q_{1} \omega\right]\right\}\right.
$$

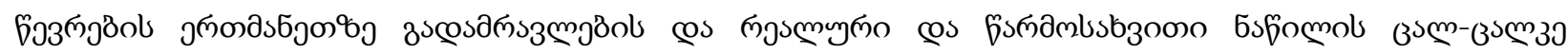

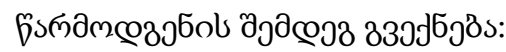

$$
A=k\left\{q_{0}\left(q_{0} k-b_{1} \omega^{2}\right)-q_{1} \omega\left[b_{2} \omega^{3}+\left(b_{0}+k q_{1} \omega\right]\right\}+j k\left\{q_{0}\left[b_{2} \omega^{3}+\left(b_{0}+k q_{1}\right) \omega+q_{1} \omega\left(q_{0} k-b_{1} \omega^{2}\right)\right\},\right.\right.
$$

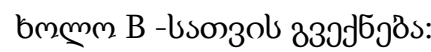

$$
B=\left(q_{0} k-b_{1} \omega^{2}\right)^{2}+\left[b_{2} \omega^{3}+\left(b_{0}+k q_{1}\right) \omega\right]^{2}
$$

ajusàsaobsce

$$
\begin{aligned}
W_{c l}(j \omega) & =\frac{A}{B}=\frac{k q_{0}\left(q_{0} k-b_{1} \omega^{2}\right)-k q_{1} \omega\left[b_{2} \omega^{3}+\left(b_{0}+k q_{1}\right) \omega\right]}{B}+ \\
+ & \frac{q_{0} k\left[b_{2} \omega^{3}+\left(b_{0}+k q_{1)}\right) \omega+q_{1} \omega\left(q_{0} k-b_{1} \omega^{2}\right]\right.}{B}
\end{aligned}
$$

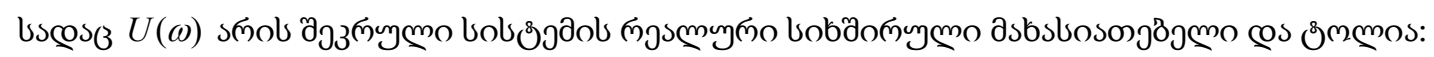




$$
U(\omega)=\frac{k q_{0}\left(q_{0} k-b_{1} \omega^{2}\right)-k q_{1} \omega\left[b_{2} \omega^{3}+\left(b_{0}+k q_{1}\right) \omega\right]}{B},
$$

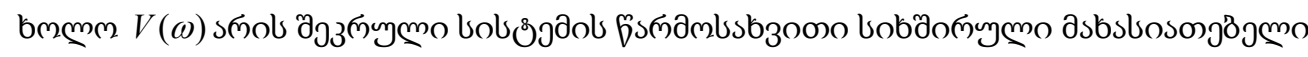

$$
V(\omega)=j \frac{\left[b_{2} \omega^{3}+\left(b_{0}+k q_{1)}\right) \omega+q_{1} \omega\left(q_{0} k-b_{1} \omega^{2}\right]\right.}{B} .
$$

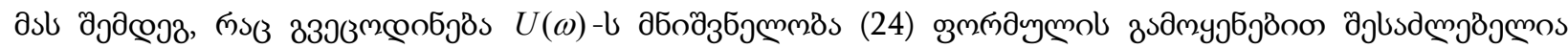

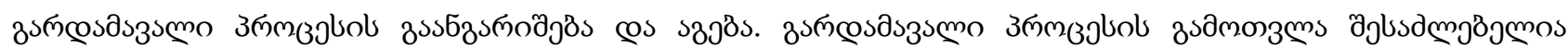

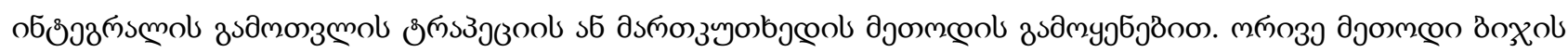

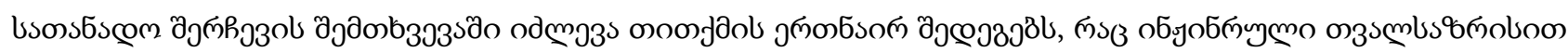

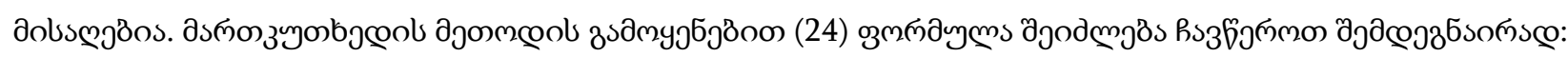

$$
\begin{gathered}
y\left(t_{1}\right)=\frac{2}{\pi} \Delta \omega\left[\frac{U\left(\omega_{1}\right)}{\omega_{1}} \operatorname{Sin}\left(t_{1} \omega_{1}\right)+\frac{U\left(\omega_{2}\right)}{\omega_{2}} \operatorname{Sin}\left(t_{1} \omega_{2}\right)+\ldots,+\frac{U\left(\omega_{l-l}\right)}{\omega_{l-1}} \operatorname{Sin}\left(t_{1} \omega_{l-1}\right)+\frac{U\left(\omega_{l}\right)}{\omega_{l}} \operatorname{Sin}\left(t_{1} \omega_{l}\right)\right]= \\
=\frac{2}{\pi} \Delta \omega\left\{\sum_{i=1}^{l} \frac{U\left(\omega_{i}\right)}{\omega_{i}} \operatorname{Sin}\left(t_{1} \omega_{i}\right)\right\} .
\end{gathered}
$$

әјдмзоммо змбобзбь:

$$
\frac{U(\omega)}{\omega} \operatorname{Sin}(\omega t)=z
$$

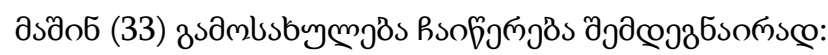

$$
y\left(t_{i}\right)=\frac{2}{\pi} \Delta \omega\left[\sum_{i=1}^{l} z_{i j}, j=1,2, \ldots, M,\right.
$$

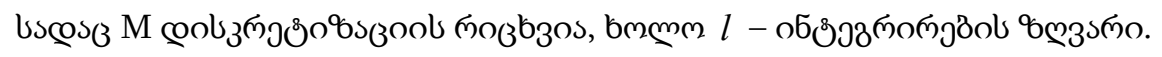

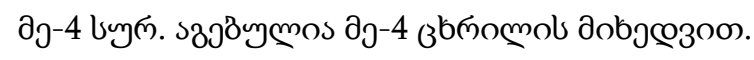

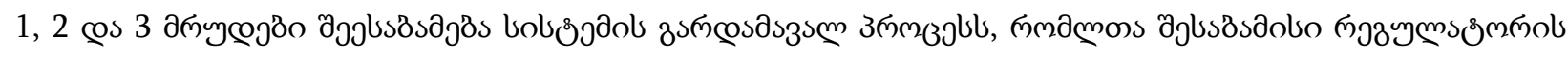
зsলs

(3mogo(3os 22) $I_{0 \min }^{(\min )}=8.40432 E-05, m=0.205,(\psi=0.724013), \omega=23.636558, q_{0}=0.719565, q_{1}=2.300645$;

(3m8\%о이 25)

$$
I_{0 \min }^{(\text {Average })}=0.010936472, m=0.22,(\psi=0.748824), \omega=21.8877, q_{0}=0.7421, q_{1}=1.983277 ;
$$

(3m8\%овоs 48)

$$
I_{0 \min }^{(\max )}=0.011378417, m=0.335,(\psi=0.878008), \omega=13.78847, q_{0}=0.644297, q_{1}=0.833981 .
$$

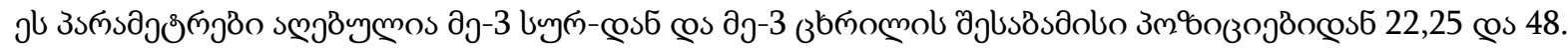

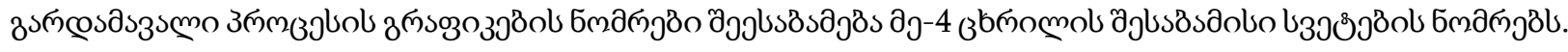




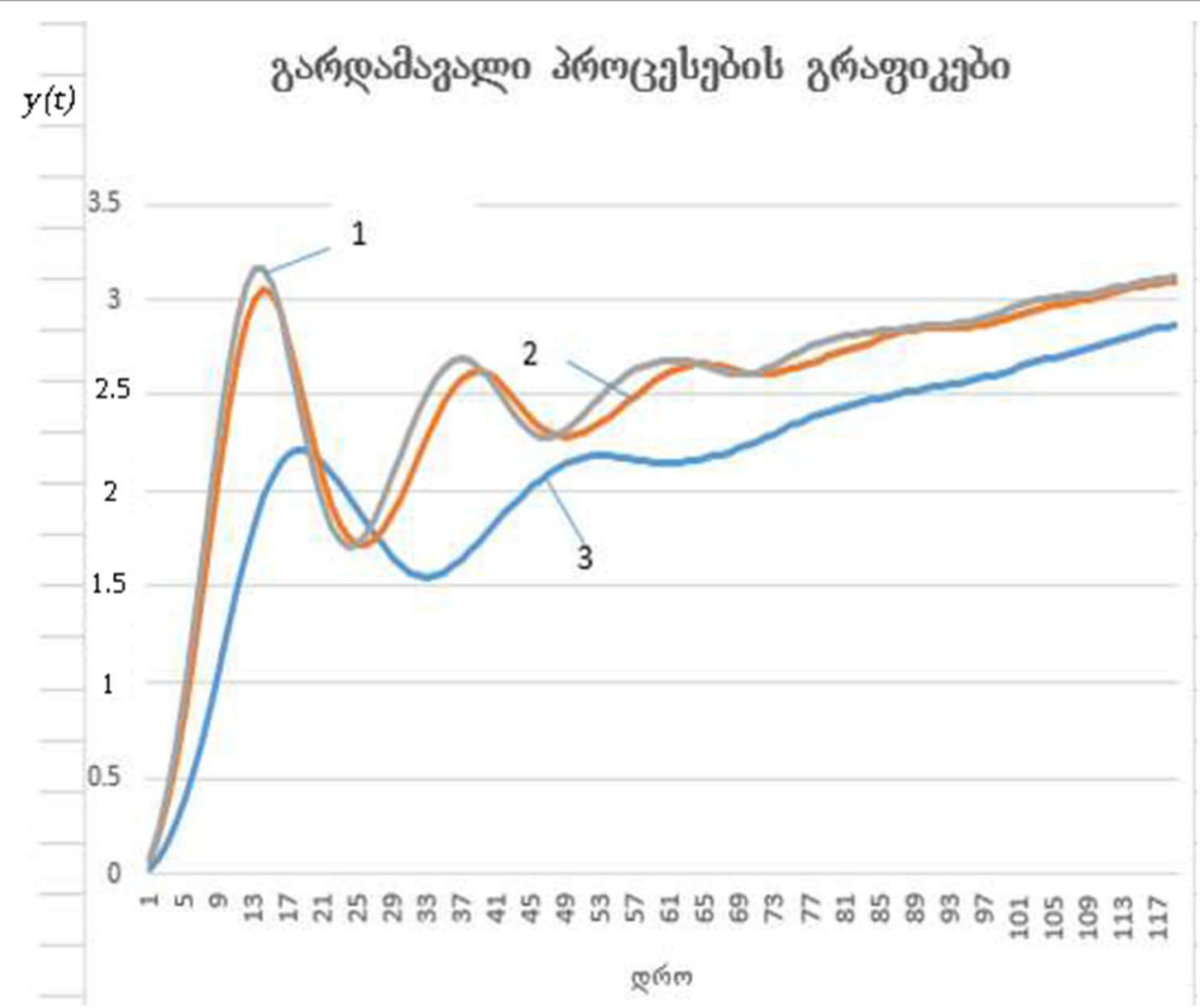

เมตฺ. 4

ob̆омо 4

\begin{tabular}{|c|c|c|c|}
\hline $\mathrm{t}$ & IominMax(3) & IominAve. (2) & IominMin (1) \\
\hline 0.01 & 0.031539086 & 0.073456233 & 0.085662666 \\
\hline 0.02 & 0.086815029 & 0.200156338 & 0.232658391 \\
\hline 0.03 & 0.163946441 & 0.372354432 & 0.430746728 \\
\hline 0.04 & 0.26379048 & 0.587820617 & 0.675905072 \\
\hline 0.05 & 0.387603327 & 0.844739048 & 1.289863455 \\
\hline 0.06 & 0.535442442 & 1.138320231 & 1.639709772 \\
\hline 0.07 & 0.705256866 & 1.459061774 & 2.339724476 \\
\hline 0.08 & 0.89260617 & 1.792538875 & 2.645928874 \\
\hline 0.09 & 1.090915308 & 2.120490809 & 2.89417791 \\
\hline 0.1 & 1.292132 & 2.422845117 & 3.067718977 \\
\hline 0.11 & 1.487621579 & 2.680229587 & 3.156562775 \\
\hline 0.12 & 1.669123059 & 2.876490509 & 3.15884397 \\
\hline 0.13 & 1.829603135 & 3.000775683 & 3.081010264 \\
\hline 0.14 & 1.963881295 & 3.0488478 & 3.023446692 \\
\hline 0.15 & 2.068952092 & & \\
\hline
\end{tabular}




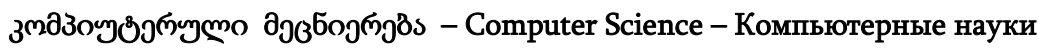

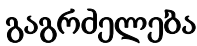

\begin{tabular}{|c|c|c|c|}
\hline 0.16 & 2.143990801 & 2.933692646 & 2.936850261 \\
\hline 0.17 & 2.190084204 & 2.793683799 & 2.745565668 \\
\hline 0.18 & 2.209770524 & 2.620561789 & 2.529239831 \\
\hline 0.19 & 2.206495133 & 2.432383501 & 2.310128183 \\
\hline 0.2 & 2.184087319 & 2.246134315 & 2.108189487 \\
\hline 0.21 & 2.146343939 & 2.076157462 & 1.939197186 \\
\hline 0.22 & 2.096770086 & 1.933169978 & 1.813638355 \\
\hline 0.23 & 2.038486853 & 1.823913471 & 1.736452627 \\
\hline 0.24 & 1.974278765 & 1.751371315 & 1.707516601 \\
\hline 0.25 & 1.906726 & 1.715396378 & 1.722667206 \\
\hline 0.26 & 1.838355248 & 1.713547328 & 1.774999064 \\
\hline 0.27 & 1.771747528 & 1.741933416 & 1.856170156 \\
\hline 0.28 & 1.709559663 & 1.795909457 & 1.957501533 \\
\hline 0.29 & 1.65444389 & 1.87053247 & 2.070743431 \\
\hline 0.3 & 1.60887803 & 1.960769911 & 2.188478012 \\
\hline 0.31 & 1.574943803 & 2.061517063 & 2.30421855 \\
\hline 0.32 & 1.554104048 & 2.16752626 & 2.412323476 \\
\hline 0.33 & 1.547031036 & 2.273361179 & 2.507864586 \\
\hline 0.34 & 1.553527263 & 2.373469429 & 2.58657028 \\
\hline 0.35 & 1.572559061 & 2.462421245 & 2.644914801 \\
\hline 0.36 & 1.602400011 & 2.535304669 & 2.680359835 \\
\hline 0.37 & 1.640857322 & 2.588214944 & 2.691691737 \\
\hline 0.38 & 1.685538611 & 2.618738072 & 2.679354393 \\
\hline 0.39 & 1.734110598 & 2.626317144 & 2.645661188 \\
\hline 0.4 & 1.784505184 & 2.612406506 & 2.594787998 \\
\hline 0.41 & 1.835042966 & 2.580358943 & 2.532492818 \\
\hline 0.42 & 1.884463968 & 2.535044292 & 2.465568678 \\
\hline 0.43 & 1.931875406 & 2.48225382 & 2.401098283 \\
\hline 0.44 & 1.976643871 & 2.427987725 & 2.345627066 \\
\hline 0.45 & 2.018268769 & 2.377746319 & 2.304394525 \\
\hline 0.46 & 2.056273967 & 2.335942478 & 2.280756807 \\
\hline 0.47 & 2.090146929 & 2.305525338 & 2.275897746 \\
\hline 0.48 & 2.119339477 & 2.287860847 & 2.288869858 \\
\hline 0.49 & 2.143327514 & 2.282861987 & 2.316942751 \\
\hline 0.5 & 2.161711392 & 2.289314619 & 2.356179821 \\
\hline 0.51 & 2.174327894 & 2.30531099 & 2.402124681 \\
\hline
\end{tabular}




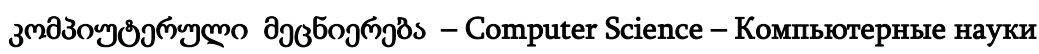

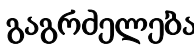

\begin{tabular}{|c|c|c|c|}
\hline 0.52 & 2.181342331 & 2.32869175 & 2.450467059 \\
\hline 0.53 & 2.183293426 & 2.357406811 & 2.497572284 \\
\hline 0.54 & 2.18107586 & 2.389735325 & 2.540797405 \\
\hline 0.55 & 2.175859319 & 2.424344304 & 2.578568134 \\
\hline 0.56 & 2.168958334 & 2.46020727 & 2.610243928 \\
\hline 0.57 & 2.161678032 & 2.496436342 & 2.635841056 \\
\hline 0.58 & 2.155166865 & 2.532098073 & 2.655705537 \\
\hline 0.59 & 2.150303725 & 2.566081807 & 2.670228308 \\
\hline 0.6 & 2.147640826 & 2.597069515 & 2.679672311 \\
\hline 0.61 & 2.147408764 & 2.623625749 & 2.684143524 \\
\hline 0.62 & 2.149577884 & 2.644390375 & 2.683696409 \\
\hline 0.63 & 2.153956929 & 2.658328645 & 2.678526128 \\
\hline 0.64 & 2.160303489 & 2.664974045 & 2.669177204 \\
\hline 0.65 & 2.168419713 & 2.664599554 & 2.65669496 \\
\hline 0.66 & 2.178211638 & 2.658266783 & 2.642660957 \\
\hline 0.67 & 2.189700665 & 2.647731264 & 2.629085361 \\
\hline 0.68 & 2.202988484 & 2.635214134 & 2.618166801 \\
\hline 0.69 & 2.218187662 & 2.623082271 & 2.611964911 \\
\hline 0.7 & 2.235338326 & 2.613499578 & 2.612056202 \\
\hline 0.71 & 2.254334697 & 2.60811879 & 2.619250156 \\
\hline 0.72 & 2.274881907 & 2.607874691 & 2.633431642 \\
\hline 0.73 & 2.296496055 & 2.612915767 & 2.653568576 \\
\hline 0.74 & 2.318549065 & 2.622682122 & 2.677886961 \\
\hline 0.75 & 2.34034987 & 2.636104696 & 2.70417928 \\
\hline 0.76 & 2.361243849 & 2.651876411 & 2.730182522 \\
\hline 0.77 & 2.380708623 & 2.668733102 & 2.753948317 \\
\hline 0.78 & 2.398425205 & 2.68568308 & 2.774130846 \\
\hline 0.79 & 2.414309054 & 2.702139756 & 2.790138452 \\
\hline 0.8 & 2.428495449 & 2.717937404 & 2.802125742 \\
\hline 0.81 & 2.441284034 & 2.733237561 & 2.810839933 \\
\hline 0.82 & 2.453055728 & 2.748360592 & 2.817365076 \\
\hline 0.83 & 2.464182733 & 2.763591399 & 2.822829263 \\
\hline 0.84 & 2.474951498 & 2.779013514 & 2.828143529 \\
\hline 0.85 & 2.485515246 & 2.794414996 & 2.833830075 \\
\hline 0.86 & 2.495886131 & 2.80929033 & 2.83997275 \\
\hline 0.87 & 2.505965457 & 2.822937282 & 2.846291525 \\
\hline
\end{tabular}




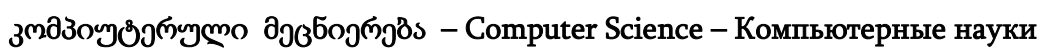

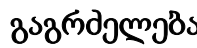

\begin{tabular}{|c|c|c|c|}
\hline 0.88 & 2.51560297 & 2.834623013 & 2.852312608 \\
\hline 0.89 & 2.524668752 & 2.843776612 & 2.857583721 \\
\hline 0.9 & 2.53311874 & 2.850158532 & 2.861874973 \\
\hline 0.91 & 2.541037105 & 2.853962491 & 2.865311126 \\
\hline 0.92 & 2.5486448 & 2.85582252 & 2.868400485 \\
\hline 0.93 & 2.556271878 & 2.856720453 & 2.871952357 \\
\hline 0.94 & 2.564300226 & 2.857812393 & 2.876903322 \\
\hline 0.95 & 2.573090268 & 2.860213608 & 2.884095542 \\
\hline 0.96 & 2.582909373 & 2.864789247 & 2.894062962 \\
\hline 0.97 & 2.59387874 & 2.871998185 & 2.906878346 \\
\hline 0.98 & 2.605951207 & 2.881823899 & 2.922101238 \\
\hline 0.99 & 2.618925094 & 2.89380664 & 2.938840801 \\
\hline 1 & 2.6324904 & 2.90716693 & 2.955921176 \\
\hline 1.01 & 2.646296358 & 2.920990191 & 2.972112899 \\
\hline 1.02 & 2.66002451 & 2.934429249 & 2.986377183 \\
\hline 1.03 & 2.673450572 & 2.946878076 & 2.998068044 \\
\hline 1.04 & 2.686480949 & 2.958078436 & 3.00704768 \\
\hline 1.05 & 2.6991568 & 2.968138508 & 3.013689608 \\
\hline 1.06 & 2.711625671 & 2.977462106 & 3.018771855 \\
\hline 1.07 & 2.724088582 & 2.986611325 & 3.023287147 \\
\hline 1.08 & 2.736736835 & 2.996137177 & 3.028215002 \\
\hline 1.09 & 2.74969282 & 3.006421524 & 3.034309707 \\
\hline 1.1 & 2.762971336 & 3.017569488 & 3.041951088 \\
\hline 1.11 & 2.776468387 & 3.029376857 & 3.051091357 \\
\hline 1.12 & 2.789981685 & 3.041380151 & 3.061305423 \\
\hline 1.13 & 2.803255932 & 3.052974658 & 3.071929199 \\
\hline 1.14 & 2.816043087 & 3.063570886 & 3.082248968 \\
\hline 1.15 & 2.828161364 & 3.07275007 & 3.091694632 \\
\hline 1.16 & 2.83953998 & 3.080379852 & 3.09998897 \\
\hline 1.17 & 2.850238332 & 3.086662243 & 3.107217766 \\
\hline 1.18 & 2.860434718 & 3.092101255 & 3.113805212 \\
\hline \multirow[t]{2}{*}{1.19} & 2.870388309 & 3.097397697 & 3.120402124 \\
\hline & 2.870388309 & 3.097397697 & 3.15884397 \\
\hline
\end{tabular}




\section{cosb $33^{6 s}$}

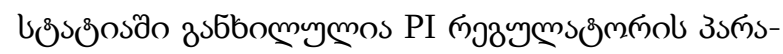

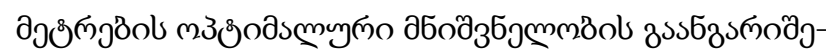

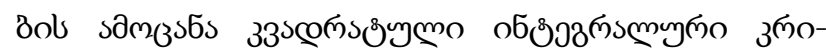

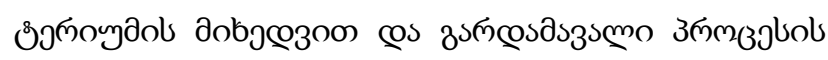

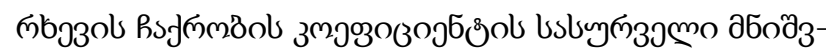

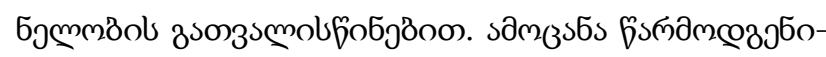

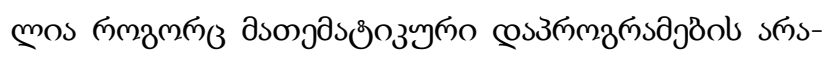
бпозозо замззьь.

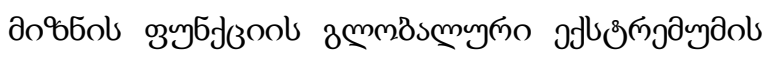

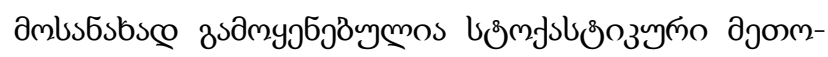

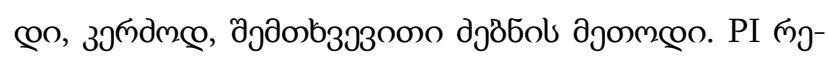

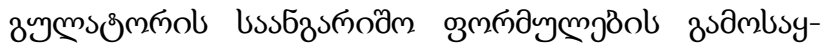

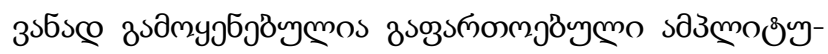

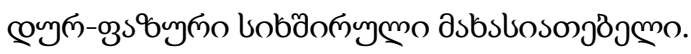

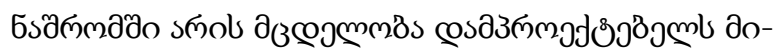

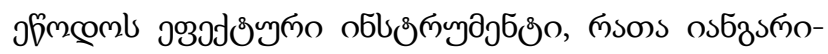

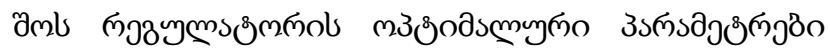

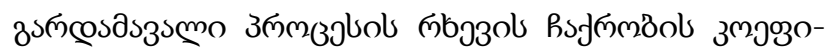
зоэбоol usbyn̆

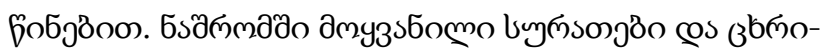

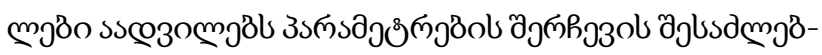

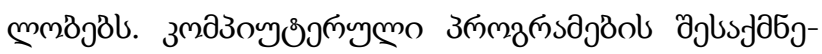

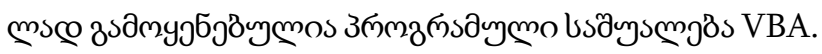

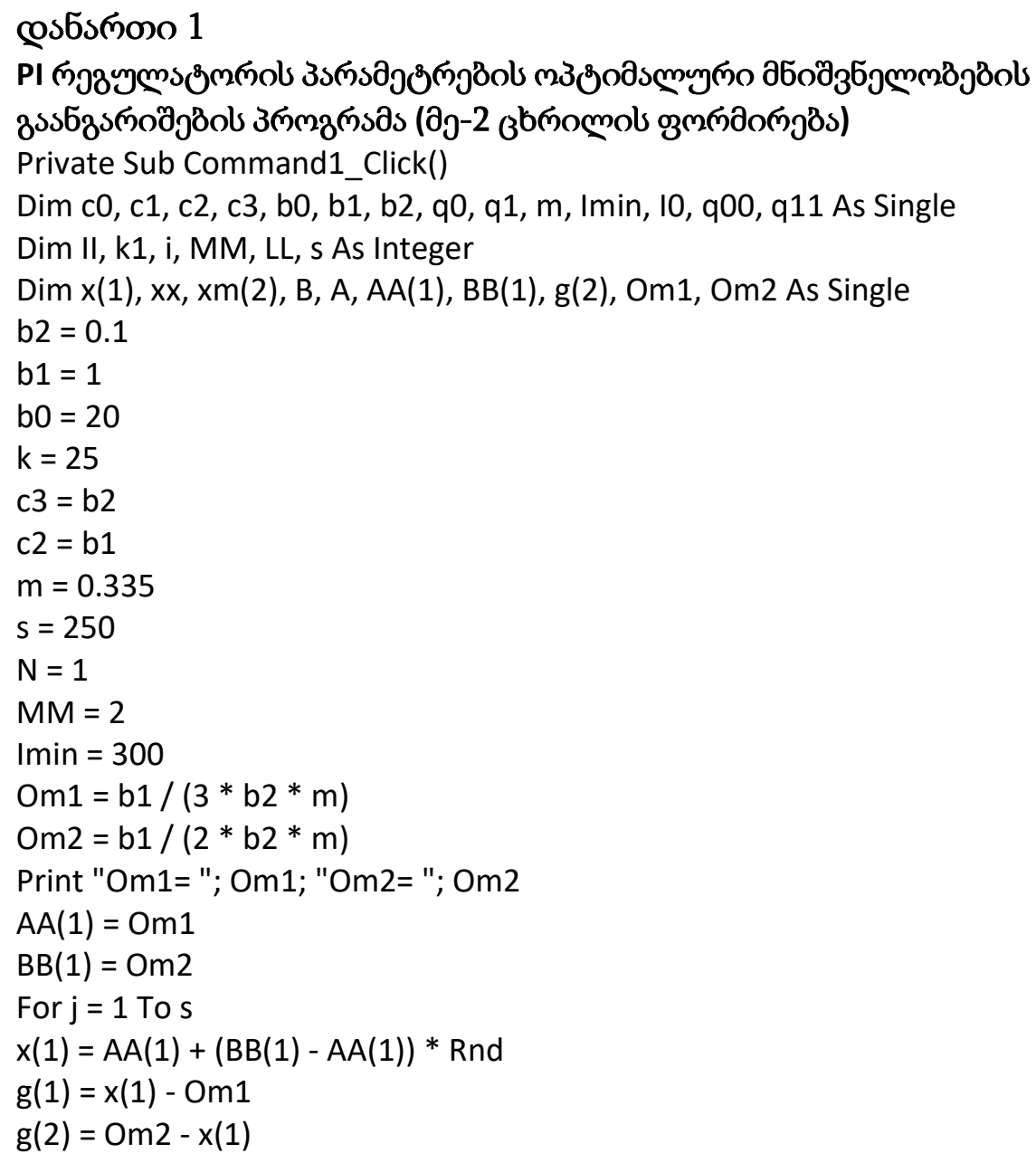




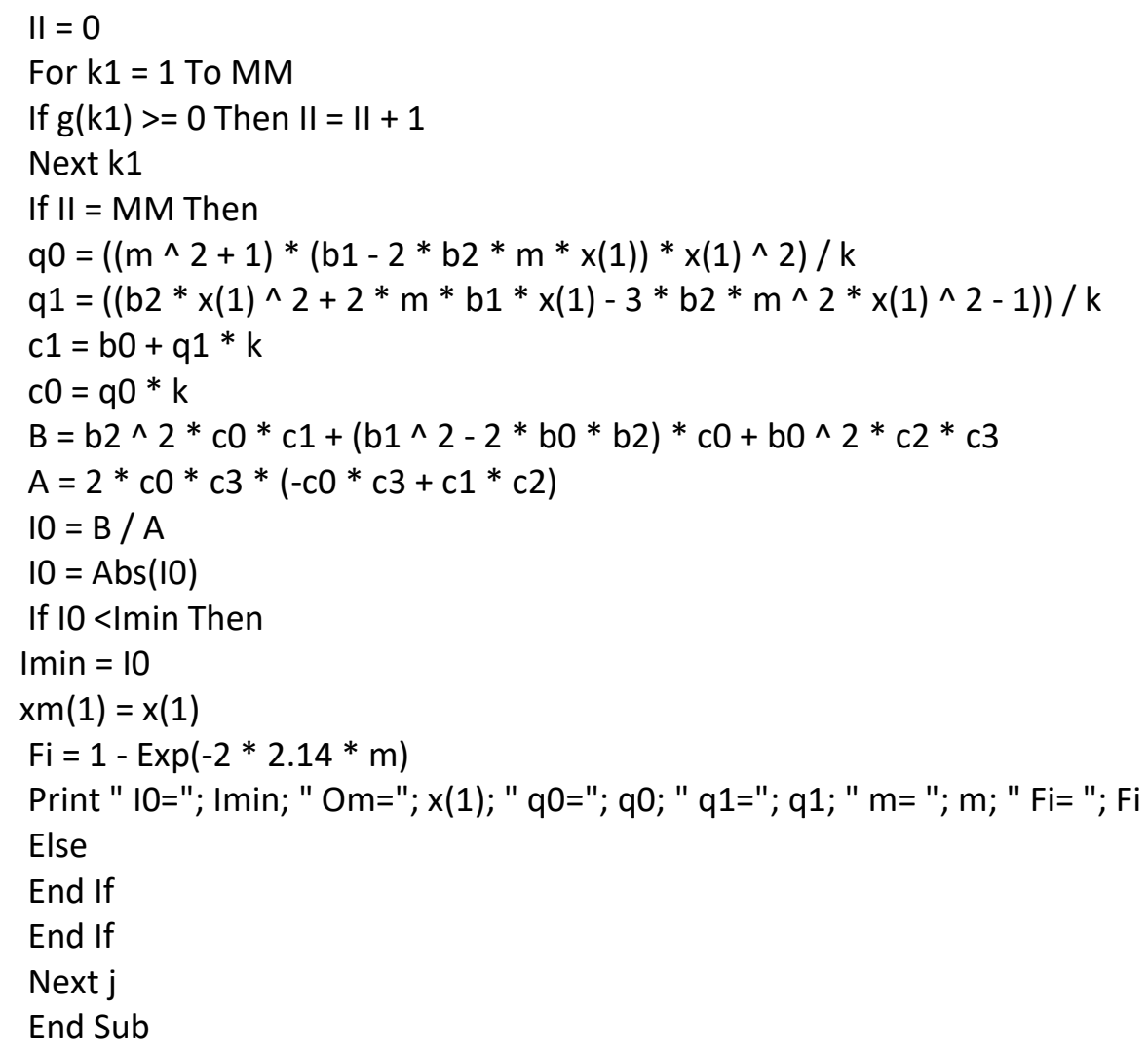




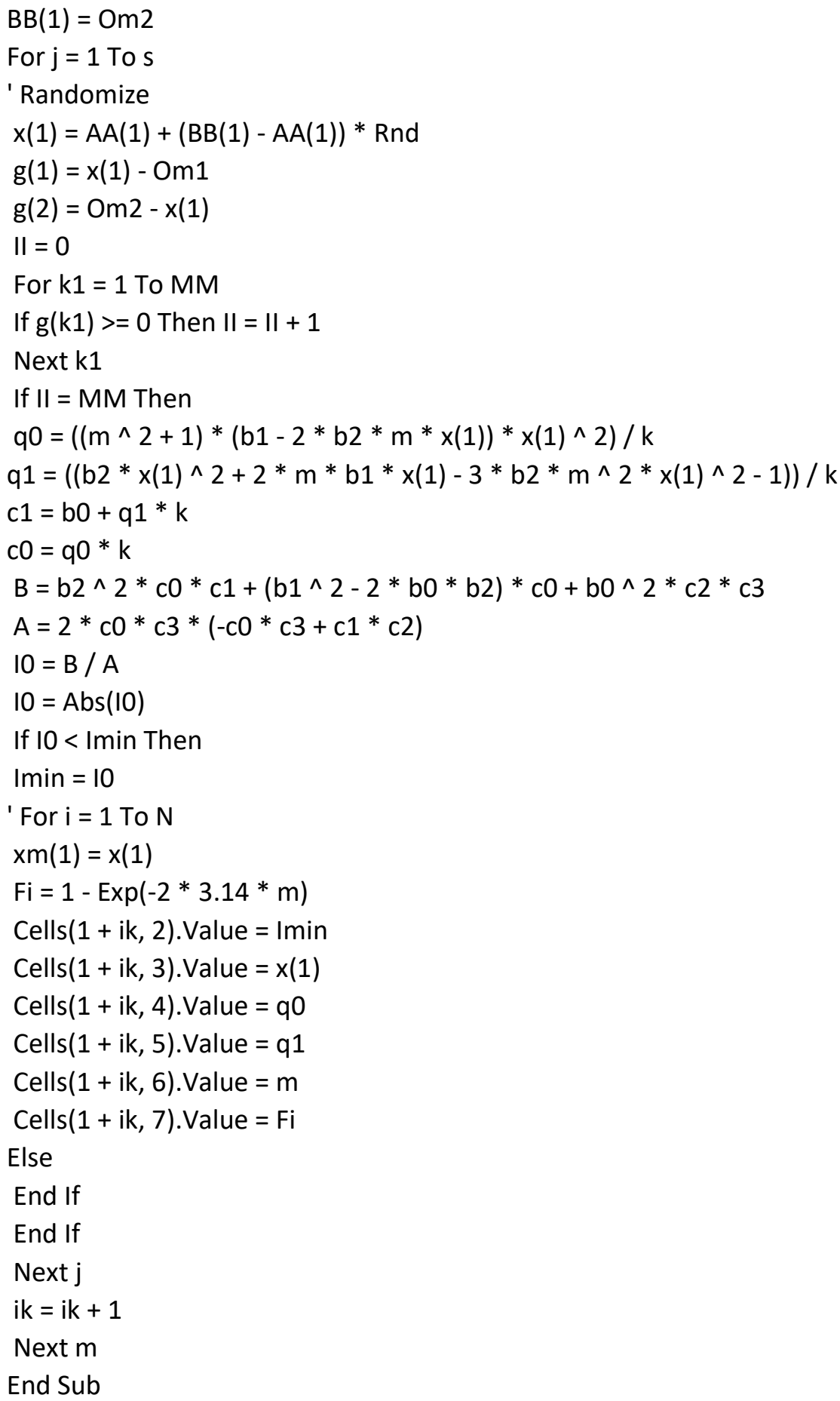




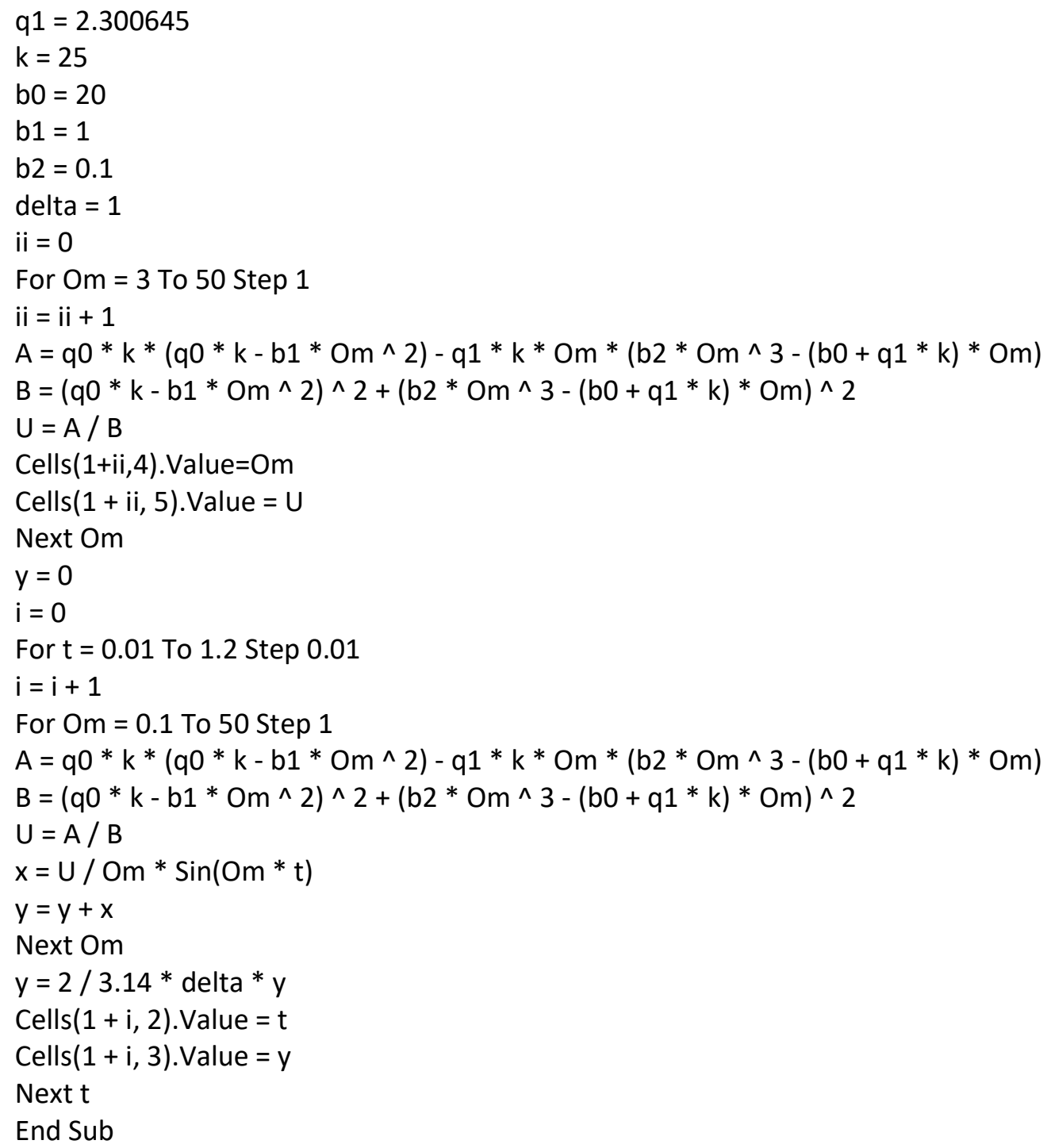

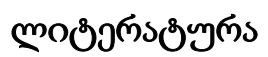

1. E. Stephani. Basics of calculating the setting of regulators of heat and power processes (Edition 2), 2014. (In Russian);

2. O. Prokhorova, S. Neklyudov. Application of nonlinear programming problems in the simulation of ACS with real objects. Technical sciences, 2016. (In Russian);

3. S. Novikov. Optimization of automatic control systems of heat and power equipment Part 1. Methods for determining the optimal parameters of settings of control devices. Tutorial. - Novosibirsk, NSTU, 2006 . 108 p. (In Russian);

4. E. Dudnikov. Bases of automatic regulation of thermal processes. \"Gosenergoizdat \", 1956. (In Russian);

5. Theory of automatic control. Ed. A.V. Has not extinguished. Textbook for universities. Ed. 2nd rev. M. 1956. (In Russian). 


\title{
UDC 62-52
}

SCOPUS CODE 1701

https://doi.org/10.36073/1512-0996-2021-1-75-97

\section{Determination of the Optimal Values of the Parameters of the PI Controller by Minimum Integral Quadratic Criterion Taking Into Account the Values Transient Damping Coefficient}

\author{
Badri Gvasalia Department of Computer-Aided Design of Construction, Georgian Technical \\ University, Georgia, 0160, Tbilisi, 68 ${ }^{\mathrm{B}}$ M. Kostava srt. \\ E-mail: Gvasaliabadri01@gtu.ge
}

\section{Reviewers:}

K. Odisharia, Associate Professor, Faculty of Informatics and Control Systems, GTU

E-mail: O_korneli@yahoo.com

E. Abramidze, Associate Professor, Faculty of Construction, GTU

E-mail: Edisoni.abramidze@mail.ru

Abstract. The problem of determining the optimal the values of the parameters of the PI controller by the quadratic integral criterion taking into account the coefficient of attenuation of the transition process. The problem is presented as a nonlinear mathematical problem programming. Finding the global minimum of the objective function carried out by random search, and to determine the parameters of the PI controller used the expanded amplitude-phase frequency response of the system. An attempt is made to provide designers with an effective tool for solving the above problem. The given graphs and tables facilitate the selection of parameters. To compile a computer program, the visual programming system VBA (Visual Basic for Application) is used. Concrete examples are given.

Key words: integral quadratic criterion; optimal parameters; proportional-integral controller; synthesis of an automatic control system. 


\title{
UDC 62-52
}

SCOPUS CODE 1701

https://doi.org/10.36073/1512-0996-2021-1-75-97

\section{Определение оптимальных значений параметров ПИ регулятора по минимуму интегральному квадратичному критерию с учетом значений коэффициента затухания колебаний переходного процесса}

Бадри Гвасалия Департамент компьютерного проектирования строительства, Грузинский технический университет, Грузия, 0160, Тбилиси ул. М. Костава, $68^{6}$

E-mail: b.gvasalia@gtu.ge

\section{Рецензенты:}

К. Одишария, ассоциированный профессор факультета информати и систем управления ГТУ

E-mail: O_korneli@yahoo.com

Е. Абрамидзе, ассоциированный профессор строительного факультета ГТУ

E-mail: Edisoni.abramidze@mail.ru

Аннотация. Во многих практических случаях, когда интегральный критерий принимает минимальное значение, переходный процесс системы имеет достаточно большие колебания, что недопустимо по различным техническим соображениям. Поэтому целесообразно использовать интегральный квадратичный критерий не изолированно, а в сочетании с коэффициентом флуктуации переходного процесса. Необходимость введения этого коэффициента смещает задачу к задаче многокритериальной оптимизации. В статье рассматривается задача определения оптимальных значений параметров ПИ регулятора по квадратичному интегральному критерию с учетом коэффициента степени затухания переходного процесса.

Задача представлена в виде задачи нелинейного математического программирования. Нахождение глобального минимума целевой функции осуществляется методом случайного поиска, а для определения параметров ПИ регулятора использована расширенная амплитудно-фазовая частотная характеристика системы.

В работе сделана попытка снабдить проектировщиков эффективным инструментом для решения вышеуказанной задачи. Приведенные чертежи и таблицы облегчают выбор параметров. Для составления компьютерной программы используется система визуального программирования VBA (visual basic for application)

Ключевые слова: оптимальные параметры; пропорционально-интегральный регулятор; синтез системы автоматического управления; интегральный квадратичный критерий.

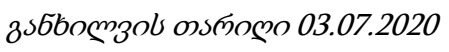

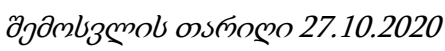

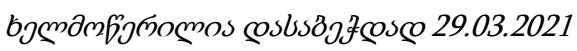

